\title{
Intensity-based Valuation of Residential Mortgages: an Analytically Tractable Model*
}

\author{
Vyacheslav Gorovoy ${ }^{\dagger} \quad$ Vadim Linetsky ${ }^{\ddagger}$
}

June 20, 2006

First Version: October 2005

Final Version: June 2006

To appear in Mathematical Finance

\begin{abstract}
This paper presents an analytically tractable valuation model for residential mortgages. The random mortgage prepayment time is assumed to have an intensity process of the form $h_{t}=h_{0}(t)+\gamma\left(k-r_{t}\right)^{+}$, where $h_{0}(t)$ is a deterministic function of time, $r_{t}$ is the short rate, and $\gamma$ and $k$ are scalar parameters. The first term models exogenous prepayment independent of interest rates (e.g., a multiple of the PSA prepayment function). The second term models refinancing due to declining interest rates and is proportional to the positive part of the distance between a constant threshold level and the current short rate. When the short rate follows a CIR diffusion, we are able to solve the model analytically and find explicit expressions for the present value of the mortgage contract, its principal-only and interest-only parts, as well as their deltas. Mortgage rates at origination are found by solving a non-linear equation. Our solution method is based on explicitly constructing an eigenfunction expansion of the pricing semigroup, a Feynman-Kac semigroup of the CIR diffusion killed at an additive functional that is a linear combination of the integral of the CIR process and an area below a constant threshold and above the process sample path (the so-called area functional). A sensitivity analysis of the term structure of mortgage rates and calibration of the model to market data are presented.
\end{abstract}

Keywords: Mortgage, Prepayment Intensity, Hazard Process, CIR Diffusion, Pricing Semigroup, Area Functional, Eigenfunction Expansion

\footnotetext{
${ }^{*}$ This research was supported by the National Science Foundation under grants DMI-0200429 and DMI0422937.

${ }^{\dagger}$ Quantitative Research, UBS AG, 677 Washington Boulevard, Stamford, CT 06901. This work was performed while V.G. was a doctoral student at Northwestern.

${ }^{\ddagger}$ Department of Industrial Engineering and Management Sciences, McCormick School of Engineering and Applied Sciences, Northwestern University, 2145 Sheridan Road, Evanston, IL 60208, Phone: (847) 491-2084, E-mail: linetsky@iems.northwestern.edu, Web: http://users.iems.northwestern.edu/ linetsky.
} 


\section{Introduction}

The residential mortgage market is currently the biggest segment of the U.S. fixed income markets. The total notional amount of Agency mortgage-backed securities alone, not including unsecuritized mortgage loans held by financial institutions directly and non-Agency mortgagebacked securities, currently exceeds the notional amount of publicly-traded U.S. Treasury debt. The problem of mortgage valuation is thus of significant practical importance.

A fully-amortizing fixed-rate mortgage is an annuity with fixed monthly payments. The complicating feature is that most residential mortgages allow prepayment of principal at par at any time prior to maturity, typically without any penalty. Assuming the mortgagor behaves rationally, the problem is to determine the optimal refinancing strategy: should the mortgagor refinance now, i.e., pay off the remaining principal balance on the outstanding mortgage, get into a new mortgage at a lower rate, and pay some refinancing transaction costs in conjunction with taking out a new mortgage, or wait for the rates to drop further and refinance later at a lower rate? The optimal refinancing problem is complicated by the fact that the mortgagor may refinance multiple times. This is generally more complicated than the early exercise of American options, where the option exercise results in the payment of a fixed amount. In the case of refinancing, the mortgagor enters into a new prepayable mortgage, which he can refinance as well. The early literature has ignored the possibility of sequential refinancing and treated the problem of valuing mortgages as annuities with embedded American-style options. This "option-based" approach has been pioneered by Dunn and McConnell (1981a,b) (for more recent references, surveys, and discussions see Goncharov (2003), (2004), (2006), Kalotay et al. (2004), Longstaff (2005), Pliska (2005), (2006), Stanton (1995)). Sequential refinancing has recently been addressed by Pliska (2005), (2006) and Longstaff (2005), who consider multi-stage decision models. Even ignoring sequential refinancing, the option-based approach is already computationally demanding and heavily numerical. The possibility of sequential refinancing further complicates the problem. Asside from the computational difficulties, the optimality assumption is often violated in market practice. Indeed, mortgagors often prepay lower-interest mortgages in a higher interest rate environment due to a variety of reasons, such as house sale or default. On the other hand, since individual mortgagors vary widely in their financial sophistication, when interest rates do decline and offer refinancing opportunities, many mortgagors either delay their refinancing decisions significantly, or do not act at all until it is too late and interest rates move back up.

In view of these issues, the option-based approach to prepayment modeling has not been widely adopted by market practitioners in the mortgage markets. An alternative "reducedform" approach is to assume that the prepayment time is a random time governed by some hazard rate to be estimated from empirical data on the actual prepayment experiences in large mortgage pools. Early important references on the empirical hazard rate approach are Schwartz and Torous (1989), (1992), (1993) (see Deng et al. (2000) and Kau et al. (2004) for more recent work in this direction). In this approach, historical prepayment data are used to statistically estimate the prepayment hazard rate function. This hazard rate is then used to simulate mortgage prepayments along simulated interest rate paths. The mortgage is then valued by discounting the resulting cash flows and averaging across the simulated interest rate paths. So far references on the prepayment modeling within the hazard rate framework have been largely empirical and numerical in nature, employing Monte Carlo simulation. Until recently, little work has been published on the theoretical and mathematical foundations of mortgage prepayment 
modeling in the intensity-based framework. This situation is in stark contrast to the burgeoning mathematical finance literature on the intensity-based credit risk modeling that has enjoyed remarkable development in the past decade (see, e.g., Jarrow and Turnbull (1995), Duffie and Singleton (1999), Elliot et al. (2000), as well as recent monographs Bielecki and Rutkowski (2002), Duffie and Singleton (2003), Jeanblanc et al. (2006), and Lando (2004) for surveys). Furthermore, the credit risk modeling technology has also been applied to other situations, such as the early exercise modeling and valuation of executive stock options (e.g., Carr and Linetsky (2000)).

Indeed, mortgage prepayment events are similar to defaults in the credit risk parlance. The prepayment can be seen as a "default" on the mortgage contract with recovery paid at the time of "default" and equal to the mortgage principal amount outstanding at the time. Thus, the recent advances in the intensity-based credit risk modeling can be applied to mortgage modeling and valuation. However, the mathematical finance literature on the subject of applying stochastic analysis of hazard process-based methods to mortgage valuation seems to be limited so far. Notable references include Goncharov (2003), (2004), (2006), Pliska (2005), (2006) and Kagraoka (2002). Goncharov aims at unifying the option-theoretic and reduced-form approaches and, in particular, working in continuous-time credit risk-style hazard process-based framework, derives expressions for the mortgage value function, the PDE it satisfies, and a non-linear equation for the mortgage rate. Pliska (2005), (2006) develops intensity-based mortgage valuation results in discrete time. Kagraoka (2002) models the prepayment process of a mortgage pool by point processes. Jarrow et al. (2005), while primarily focused on corporate defaults, theoretically justify the market practice of using the same prepayment intensity under both the physical measure and the risk-neutral measure, by assuming that the prepayment event risk is diversifiable.

The present paper develops a simple analytically tractable mortgage valuation model within the intensity-based approach. The random mortgage prepayment time is assumed to have an intensity process of the form $h_{t}=h_{0}(t)+\gamma\left(k-r_{t}\right)^{+}$, where $h_{0}(t)$ is a deterministic function of time, $r_{t}$ is the short rate, and $\gamma$ and $k$ are scalar parameters. The first term models exogenous prepayment independent of interest rates (e.g., a multiple of the PSA prepayment function). The second term models refinancing due to declining interest rates and is proportional to the positive part of the distance between a constant threshold level and the current short rate. When the short rate follows a CIR diffusion, we are able to solve the model analytically and find explicit expressions for the present value of the mortgage contract, its principal-only and interest-only parts, as well as their deltas measuring sensitivity of the contract value to changes in interest rates. Mortgage rates at origination are found by solving a non-linear equation. Our solution method is based on explicitly constructing an eigenfunction expansion of the pricing semigroup, a Feynman-Kac semigroup of the CIR diffusion killed at an additive functional that is a linear combination of the integral of the CIR process and an area below a constant threshold and above the process sample path (the so-called area functional). In this context, the eigenfunction expansion method appears to have several distinct advantages. First, the integrals with respect to time appearing in the general expression for the mortgage value are calculated in closed form. This leads to a simple non-linear equation for the mortgage rate that does not contain any integrals and can be solved for the mortgage rate at origination by a simple application of the Newton-Raphson iterations. Secondly, seasoned mortgage contracts, as well as their interestonly and principal-only parts, can be simply valued at some arbitrary time $t>0$ during the mortgage life (after origination). Finally, the mortgage contract delta is obtained essentially 
without any additional computational effort by directly differentiating the eigenfunctions with respect to the short rate.

The rest of the paper is organized as follows. In Section 2 we consider mortgage contracts with continuous and discrete payments without prepayment and determine mortgage rates in terms of the current term structure of interest rates at origination. The material of this section is well-known and is included to establish our notation. In Section 3 we consider mortgage contracts with continuous and discrete payments and with random prepayment in the credit risk-style intensity-based framework. In particular, we consider two problems: determining the mortgage rates at origination and valuing seasoned mortgages. This section primarily surveys known results about the intensity-based mortgage valuation following Goncharov (2003), (2004), (2006), establishes our notation, and presents some basic properties of mortgages that will be needed later in the paper. In Section 4 we introduce our model specification for the interest rate process (CIR) and the prepayment intensity Eq.(4.2). In Section 5 we solve our model. Theorem 5.1 gives a spectral representation (which in this case reduces to an eigenfunction expansion) for the Feynman-Kac semigroup of the CIR diffusion killed at an additive functional. This result generalizes the result in Gorovoi and Linetsky (2004) obtained in the context of an entirely different financial model. This result allows us to obtain explicit expressions for the non-linear equation determining the mortgage rate and the value functions of seasoned mortgages and their deltas. In Section 6 we demonstrate numerical properties of the eigenfunction expansion in this setting, develop case studies of determining mortgage rates and valuing seasoned mortgages, provide sensitivity analysis, and calculate implied prepayment intensity parameters by calibrating our model to the market data on mortgage rates. Section 7 concludes the paper. Proof of Theorem 5.1 is given in Appendix A. Appendix B contains some background on confluent hypergeometric functions used in the paper. Appendix $\mathrm{C}$ contains some results on the CIR model.

\section{Mortgage Contracts without Prepayment}

We consider a fixed-rate mortgage contract that originates at time $t=0$ and matures at time $T>0$. The borrower takes out a loan of $P_{0}$ dollars at origination and pays a continuous coupon stream at the constant rate of $c>0$ dollars per annum during the mortgage life $[0, T]$. For a mortgage originated at time $t=0$ and with maturity $T$, the interest is compounded at the contractual mortgage rate $m(0, T)$ or simply $m$ (expressed in percent per annum) fixed at origination. The remaining mortgage principal at time $t \in[0, T]$ is denoted by $P(t)$ or $P_{t}$. In the absence of prepayment, it satisfies the following ordinary differential equation (ODE) (over an infinitesimal time interval $d t$ the total payment made by the borrower, $c d t$, consists of the interest payment $m P(t) d t$ and the scheduled principal repayment $-d P(t)$, i.e. $c d t=$ $\left.m P(t) d t-d P(t), \quad P(0)=P_{0}\right)$ :

$$
\frac{d P(t)}{d t}=m P(t)-c, \quad P(0)=P_{0} .
$$

The solution is

$$
P(t)=P_{0} e^{m t}+\frac{c}{m}\left(1-e^{m t}\right)
$$


Since the mortgage is fully amortized and the remaining principal at maturity is zero, $P(T)=0$, the payment rate $c$ is determined in terms of the contractual mortgage rate $m$ :

$$
c=\frac{m P_{0}}{1-e^{-m T}},
$$

and

$$
P_{t}=P_{0} \frac{1-e^{-m(T-t)}}{1-e^{-m T}}, t \in[0, T] .
$$

In the absence of prepayment, the cash flows from the mortgage are deterministic, and the present value of the mortgage at time $t \in[0, T]$ can be calculated in terms of the current term structure of interest rates, $\{B(t, u), u \in[t, T]\}$, where $B(t, u)$ is the time- $t$ price of the $u$-maturity zero-coupon bond with unit face value, so that

$$
M(t)=c A_{c}(t, T), \text { where } A_{c}(t, T)=\int_{t}^{T} B(t, u) d u
$$

is the present value of the continuous annuity from $t$ to $T$ continuously paying at the rate of one dollar per annum. At origination, to prevent arbitrage in the mortgage origination market, the present value of the mortgage must be equal to the mortgage principal, $M(0)=P_{0}$, and $P_{0}=c A_{c}(0, T)$. Recalling $(2.1)$, the mortgage rate $m=m(0, T)$ is seen to satisfy the following non-linear equation:

$$
\frac{1}{m}\left(1-e^{-m T}\right)=A_{c}(0, T)
$$

Solving this equation determines the continuous mortgage rate at the contract origination in terms of the then-current term structure of interest rates.

In practice the mortgage payments are paid at discrete time intervals $\Delta>0$ (typically monthly, $\Delta=1 / 12$ ). The borrower makes discrete equal payments of $c \Delta>0$ dollars at times $t_{k}=k \Delta, k=1, \ldots, N, N=T / \Delta$, where $T$ is the mortgage maturity (for simplicity assume $T$ is an integer number of years away from origination; here $c$ is the annualized payment rate in dollars, so that the periodic payment at times $t_{k}$ is equal to $c \Delta$ ). The initial mortgage principal is $P_{0}$. The principal remaining immediately after the $k$ th payment is made is $P_{k \Delta}$. In the absence of prepayment, it satisfies the following recursive equation (the total payment made by the borrower of $c \Delta$ dollars at time $t_{k+1}=(k+1) \Delta$ consists of the interest payment $m \Delta P_{k \Delta}$ and the scheduled principal repayment $P_{k \Delta}-P_{(k+1) \Delta}$; here $m=m(0, T)$ is the annualized contractual mortgage rate for the $T$-maturity mortgage):

$$
P_{(k+1) \Delta}=(1+m \Delta) P_{k \Delta}-c \Delta .
$$

The solution to this recursion is

$$
P_{k \Delta}=P_{0}(1+m \Delta)^{k}-\frac{c}{m}\left((1+m \Delta)^{k}-1\right) .
$$

Since the mortgage is fully amortized and the remaining principal at maturity is zero, $P(T)=0$, the payment rate $c$ is determined in terms of the mortgage rate $m$ :

$$
c=\frac{m P_{0}}{1-(1+m \Delta)^{-N}}
$$


and

$$
P_{k \Delta}=P_{0} \frac{1-(1+m \Delta)^{-(N-k)}}{1-(1+m \Delta)^{-N}}
$$

In the absence of prepayment, the present value of the mortgage at time $t \in[0, T]$ can be calculated in terms of the current term structure of interest rates:

$$
M(t)=c A_{\Delta}(t, T), \text { where } A_{\Delta}(t, T)=\sum_{k=[t / \Delta]+1}^{N} B\left(t, t_{k}\right) \Delta
$$

is the present value of the discrete annuity paying $\Delta$ dollars at times $t_{k}$ (here $[x]$ denotes the integer part of $x$ ). At origination, $t=0$, to prevent arbitrage in the mortgage origination market, the present value of the mortgage must be equal to the mortgage principal $P_{0}$ and $P_{0}=c A_{\Delta}(0, T)$. Recalling $(2.4)$, the mortgage rate $m=m(0, T)$ is seen to satisfy the following non-linear equation:

$$
\frac{1}{m}\left(1-(1+m \Delta)^{-N}\right)=A_{\Delta}(0, T) .
$$

Solving this equation determines the discrete mortgage rate at the contract origination in terms of the then-current term structure of interest rates.

\section{$3 \quad$ Valuation of Mortgages and Determination of Mortgage Rates with Prepayment in the Intensity-based Framework}

We start with a probability space $(\Omega, \mathcal{G}, \mathbb{Q})$ carrying a standard Brownian motion $\left\{B_{t}, t \geq 0\right\}$ and an exponential random variable with unit mean $e \sim \operatorname{Exp}(1)$ and independent of the Brownian motion $B$. We denote by $\mathbb{F}=\left\{\mathcal{F}_{t}, t \geq 0\right\}$ the filtration generated by the Brownian motion. We assume frictionless markets, no arbitrage, and take an equivalent martingale measure (EMM) $\mathbb{Q}$ as given. We model the instantaneous interest rate (the short rate) under the EMM as a positive continuous process adapted to the Brownian filtration $\mathbb{F}$.

To model prepayment, we introduce a positive prepayment intensity (hazard rate) process $\left\{h_{t}, t \geq 0\right\}$ adapted to the Brownian filtration $\mathbb{F}$. We model the random time of prepayment $\tau$ as the first time when the hazard process $\int_{0}^{t} h_{u} d u$ is greater or equal to the random level $e \sim \operatorname{Exp}(1):$

$$
\tau=\inf \left\{t \geq 0: \int_{0}^{t} h_{u} d u \geq e\right\} .
$$

At time $\tau$, the borrower prepays the remaining principal balance of the mortgage $P_{\tau}$.

To keep track of how information is revealed over time, we introduce a prepayment indicator process $\left\{N_{t}, t \geq 0\right\}, N_{t}=\mathbf{1}_{\{t \geq \tau\}}$ (a one-jump process equal to zero before prepayment and jumping to one at $\tau$ ), a filtration $\mathbb{D}=\left\{\mathcal{D}_{t}, t \geq 0\right\}$ generated by $N_{t}$, and an enlarged filtration $\mathbb{G}=\left\{\mathcal{G}_{t}, t \geq 0\right\}, \mathcal{G}_{t}=\mathcal{F}_{t} \vee \mathcal{D}_{t}$. Then the compensated process $N_{t}-\int_{0}^{t \wedge \tau} h_{s} d s$ is a $(\mathbb{G}, \mathbb{Q})$ martingale.

The prepayment event is similar to the default event. Until prepayment or maturity, whichever comes first, the lender receives the cash flow stream at the rate of $c$ dollars per annum. At the time of prepayment $\tau$, the lender receives a recovery payment equal to the remaining principal $P_{\tau}$. Using the standard credit risk modeling framework, the present value at 
time $t<T$ of the mortgage with continuous payments is given by:

$$
\begin{gathered}
M_{t}=\mathbb{E}\left[\int_{t}^{T} c e^{-\int_{t}^{u} r_{s} d s} \mathbf{1}_{\{\tau>u\}} d u \mid \mathcal{G}_{t}\right]+\mathbb{E}\left[e^{-\int_{t}^{\tau} r_{s} d s} P_{\tau} \mathbf{1}_{\{t<\tau \leq T\}} \mid \mathcal{G}_{t}\right] \\
=\mathbf{1}_{\{\tau>t\}} \mathbb{E}\left[\int_{t}^{T}\left(c+h_{u} P_{u}\right) e^{-\int_{t}^{u}\left(r_{s}+h_{s}\right) d s} d u \mid \mathcal{F}_{t}\right]
\end{gathered}
$$

where the payment rate $c$ and the remaining principal balance $P_{u}$ are given by Eqs.(2.1) and (2.2), respectively. Introducing the following notation for $u \geq t \geq 0$ :

$$
\begin{aligned}
Q(t, u) & :=\mathbb{E}\left[e^{-\int_{t}^{u}\left(r_{s}+h_{s}\right) d s} \mid \mathcal{F}_{t}\right], \\
H(t, u) & :=\mathbb{E}\left[h_{u} e^{-\int_{t}^{u}\left(r_{s}+h_{s}\right) d s} \mid \mathcal{F}_{t}\right],
\end{aligned}
$$

given no prepayment by time $t$, the mortgage value can be re-written in the form

$$
\begin{gathered}
M_{t}=\int_{t}^{T}\left[c Q(t, u)+P_{u} H(t, u)\right] d u \\
=\frac{P_{0}}{1-e^{-m T}} \int_{t}^{T}\left[m Q(t, u)+\left(1-e^{-m(T-u)}\right) H(t, u)\right] d u,
\end{gathered}
$$

where we used Eqs.(2.1) and (2.2).

At time zero, to prevent arbitrage in the mortgage origination market, the mortgage present value (3.4) is equal to the initial mortgage principal, $M_{0}=P_{0}$, and we obtain a non-linear equation for the mortgage rate $m=m(0, T)$ :

$$
m \int_{0}^{T} Q(0, u) d u+\int_{0}^{T}\left(1-e^{-m(T-u)}\right) H(0, u) d u=1-e^{-m T}
$$

Eqs.(3.4) and (3.5) can be expressed in an alternative form due to Goncharov (2003), (2004), (2006). Given no prepayment by time $t \geq 0$, the value of the mortgage at time $t$ can be expressed as

$$
\begin{gathered}
M_{t}=P_{t}+\mathbb{E}\left[\int_{t}^{T}\left(m-r_{u}\right) P_{u} e^{-\int_{t}^{u}\left(r_{s}+h_{s}\right) d s} d u \mid \mathcal{F}_{t}\right] \\
=P_{t}+\int_{t}^{T} P_{u}[m Q(t, u)-R(t, u)] d u \\
=P_{t}\left\{1+\int_{t}^{T}\left(\frac{1-e^{-m(T-u)}}{1-e^{-m(T-t)}}\right)[m Q(t, u)-R(t, u)] d u\right\},
\end{gathered}
$$

where

$$
R(t, u):=\mathbb{E}\left[r_{u} e^{-\int_{t}^{u}\left(r_{s}+h_{s}\right) d s} \mid \mathcal{F}_{t}\right] .
$$

Note that the three quantities $Q(t, u), H(t, u)$ and $R(t, u)$ are related by

$$
\frac{\partial Q}{\partial u}(t, u)+H(t, u)+R(t, u)=0 .
$$


To prove that the right-hand side of Eq.(3.4) is equal to the right-hand side of Eq.(3.6), we need to prove that

$$
\int_{t}^{T}\left[\left(c-m P_{u}\right) Q(t, u)+P_{u}(H(t, u)+R(t, u))\right] d u=P_{t}
$$

To prove this equality, use Eq.(3.8), integrate by parts, recall that $d P(u) / d u=m P(u)-c$, and recall that at maturity $P_{T}=0$.

In the representation (3.6) the mortgage can be interpreted in terms of a "defaultable" swap with amortizing principal $P_{t}$. The present value of the mortgage at time $t$ is equal to the remaining principal at time $t$ plus the value of a swap where the borrower continuously pays the lender at the (fixed) mortgage rate $m$ applied to the remaining principal $P_{u}, u \in[t, T]$, while the lender pays the borrower at the current (floating) short rate $r_{u}$ applied to $P_{u}$. The swap terminates at the time of prepayment $\tau$ ("default") or maturity $T$, whichever comes first.

At origination the mortgage present value is equal to the mortgage principal, $M_{0}=P_{0}$, and, hence, the present value of the swap is zero:

$$
\mathbb{E}\left[\int_{0}^{T}\left(m-r_{u}\right) P_{u} e^{-\int_{0}^{u}\left(r_{s}+h_{s}\right) d s} d u\right]=\int_{0}^{T} P_{u}[m Q(0, u)-R(0, u)]=0 .
$$

Recalling (2.2), the mortgage rate $m$ is seen to satisfy the following non-linear equation:

$$
m=\frac{\int_{0}^{T}\left(1-e^{-m(T-u)}\right) R(0, u) d u}{\int_{0}^{T}\left(1-e^{-m(T-u)}\right) Q(0, u) d u}
$$

The mortgage rate at origination is determined by solving this equation numerically.

Given the mortgage rate $m$ fixed at origination, Eq.(3.4) or, alternatively, Eq.(3.6) determines the value of the seasoned mortgage at any time $t \in[0, T]$ during its life. Eq.(3.6) provides a convenient decomposition of the mortgage into its principal only (PO) and interest only (IO) parts:

$$
M_{t}=P O_{t}+I O_{t}
$$

where

$$
I O_{t}=\mathbb{E}\left[\int_{t}^{T} m P_{u} e^{-\int_{t}^{u}\left(r_{s}+h_{s}\right) d s} d u \mid \mathcal{F}_{t}\right]=m P_{t} \int_{t}^{T}\left(\frac{1-e^{-m(T-u)}}{1-e^{-m(T-t)}}\right) Q(t, u) d u
$$

and

$$
\begin{aligned}
& P O_{t}=P_{t}-\mathbb{E}\left[\int_{t}^{T} r_{u} P_{u} e^{-\int_{t}^{u}\left(r_{s}+h_{s}\right) d s} d u \mid \mathcal{F}_{t}\right] \\
& =P_{t}\left\{1-\int_{t}^{T}\left(\frac{1-e^{-m(T-u)}}{1-e^{-m(T-t)}}\right) R(t, u) d u\right\} .
\end{aligned}
$$

In the discrete payments case, the lender receives payments of $c \Delta$ dollars at times $k \Delta$ until prepayment or maturity, whichever comes first. At the time of prepayment $\tau$, the lender receives a recovery payment equal to the remaining principal $P_{\tau}$. Note that the prepayment is allowed at any time and not just at the discrete monthly payment dates. This is in accordance with the actual practice in the mortgage markets. Typically the lender calculates the remaining mortgage balance each day according to the accrued interest calculation, and the borrower can pay off the 
mortgage on any date. More precisely, at some time $t \in[k \Delta,(k+1) \Delta)$, the remaining principal $P_{t}$ is equal to the principal $P_{k \Delta}$ remaining at time $t_{k}=k \Delta$ after the $k$ th payment is made plus accrued interest since the $k$ th payment:

$$
P_{t}=P_{k \Delta}(1+m(t-k \Delta))
$$

where $P_{k \Delta}$ is given by Eq.(2.5). Generally, for $t \in[0, T]$ we can write

$$
P_{t}=P_{[t / \Delta] \Delta}(1+m(t-[t / \Delta] \Delta))=P_{0} \frac{1-(1+m \Delta)^{-(N-[t / \Delta])}}{1-(1+m \Delta)^{-N}}(1+m(t-[t / \Delta] \Delta)),
$$

where $[x]$ denotes the integer part of $x$.

Using the intensity-based credit risk modeling framework, the present value at time $t<T$ of the mortgage with discrete payments is given by:

$$
\begin{gathered}
M_{t}=\sum_{k=[t / \Delta]+1}^{N} c \Delta \mathbb{E}\left[e^{-\int_{t}^{k \Delta} r_{s} d s} \mathbf{1}_{\{\tau>t\}} \mid \mathcal{G}_{t}\right]+\mathbb{E}\left[e^{-\int_{t}^{\tau} r_{s} d s} P_{\tau} \mathbf{1}_{\{t<\tau \leq T\}} \mid \mathcal{G}_{t}\right] \\
=\mathbf{1}_{\{\tau>t\}} \sum_{k=[t / \Delta]+1}^{N} c \Delta \mathbb{E}\left[e^{-\int_{t}^{k \Delta}\left(r_{s}+h_{s}\right) d s} \mid \mathcal{F}_{t}\right]+\mathbf{1}_{\{\tau>t\}} \mathbb{E}\left[\int_{t}^{T} h_{u} P_{u} e^{-\int_{t}^{u}\left(r_{s}+h_{s}\right) d s} d u \mid \mathcal{F}_{t}\right],
\end{gathered}
$$

where the payment $c \Delta$ and the remaining principal balance $P_{u}$ are given by Eqs.(2.4) and (3.13), respectively. Given no prepayment by time $t$, the mortgage value can be re-written in the form

$$
M_{t}=\sum_{k=[t / \Delta]+1}^{N} c \Delta Q(t, k \Delta)+\int_{t}^{T} P_{u} H(t, u) d u
$$

where the $P_{t}$ is given by Eq.(3.13), and $Q$ and $H$ are defined in Eqs.(3.2), (3.3). The present value at time $t$ of the interest-only part of the mortgage is:

$$
I O_{t}=m \Delta \sum_{k=[t / \Delta]+1}^{N} P_{(k-1) \Delta} Q(t, k \Delta),
$$

and the principal-only part is:

$$
P O_{t}=M_{t}-I O_{t}
$$

Setting the present value of the mortgage at origination equal to the initial mortgage principal, $M_{0}=P_{0}$, and recalling Eqs.(2.4) and (3.13), we obtain a non-linear equation for the mortgage rate $m=m(0, T)$ for the mortgage contract with discrete payments:

$$
\begin{gathered}
m \Delta \sum_{k=1}^{N} Q(0, k \Delta)+\int_{0}^{T}\left(1-(1+m \Delta)^{[u / \Delta]-N}\right)(1+m(u-[u / \Delta] \Delta)) H(0, u) d u \\
=1-(1+m \Delta)^{-N} .
\end{gathered}
$$




\section{Model Specification}

We now specify the interest rate and the prepayment intensity processes. We model the short rate by the Cox-Ingersoll-Ross (CIR) diffusion process $\left\{r_{t}, t \geq 0\right\}$, a unique strong solution of the stochastic differential equation (SDE):

$$
d r_{t}=\kappa\left(\theta-r_{t}\right) d t+\sigma \sqrt{r_{t}} d B_{t}, \quad r_{0}=r>0,
$$

where $\kappa>0, \theta>0$, and $\sigma>0$ are the rate of mean reversion, the long-run interest rate level, and interest rate volatility, respectively. The process parameters are assumed to satisfy the Feller condition $2 \kappa \theta \geq \sigma^{2}$ to insure that the origin is an inaccessible boundary for the process and, thus, the process stays strictly positive.

We assume that the prepayment intensity is a function of time and the current interest rate $r_{t}$ only, $h_{t}=h\left(r_{t}, t\right)$, and adopt a simple specification for the prepayment function $h(r, t)$ :

$$
h(r, t)=h_{0}(t)+\gamma(k-r)^{+} .
$$

Here $\gamma \geq 0$ and $k>0$ are constant parameters, and $h_{0}(t)$ is a deterministic function of time. It captures exogenous prepayment due to reasons independent of the level of interest rates, such as prepayment due to sale of house or mortgage insurance payment due to default by the borrower.

We will consider two specifications for $h_{0}(t)$ : the constant prepayment rate ( $h_{0}$ is a constant) known in the industry as the CPR assumption, as well as the time-dependent PSA assumption. Making $h_{0}(t)$ a function of time allows us to account for the fact that the prepayment intensity changes with the age of the mortgage. The Public Securities Association (PSA) recommends the use of a piecewise linear prepayment intensity (e.g., Cizek et al. (2005), Section 9.3.2.1 or Kalotay et al. (2004), p.957):

$$
h_{0}(t)=b\left(a t \mathbf{1}_{\left\{t<T^{*}\right\}}+a T^{*} \mathbf{1}_{\left\{t \geq T^{*}\right\}}\right) .
$$

According to the PSA, the parameters are $a=0.024$ and $T^{*}=30 / 12=2.5$. For $b=1$, this implies that the prepayment intensity starts from zero at origination, grows by $0.2 \%$ per month during the first 30 months, and is equal to $6 \%$ per annum thereafter. This curve is accepted by the market practitioners as a benchmark prepayment intensity. It is known as the $100 \%$ PSA curve. The parameter $b>0$ sets the desired translation level of this benchmark. For example, if $b=0.75$, the prepayment intensity is $75 \%$ of the PSA level. The time-independent exogenous intensity $h_{0}$ under the CPR assumption corresponds to neglecting the initial ramping-up of the PSA intensity and considering a constant intensity over the entire life of the mortgage.

Note that the exogenous prepayment is not necessarily detrimental to the lender. Since it is independent of interest rates, the prepayment may happen when the current rate is higher than the rate at origination, in which case the lender can re-lend the principal it receives due to prepayment at the currently prevailing higher rate. Of course, if the prepayment happens when the current rate is lower, the lender will only be able to re-lend the principal at the lower rate.

The second term in (4.2) captures the endogenous prepayment due to refinancing due to interest rate declines. ${ }^{1}$ The threshold $k>0$ in our specification would typically be set equal to the prevailing short rate at origination, $k=r_{0}$, or, more precisely, a lower value $k=r_{0}-s$, where the spread $s \geq 0$ accounts for refinancing transaction costs, and $\gamma \geq 0$ is the constant intensity

\footnotetext{
${ }^{1}$ The specification for the prepayment rate similar to our $\gamma(k-r)^{+}$was early discussed in Duffie (1996), p.149.
} 
scale parameter. This specification suggests that a decline in the market interest rate leads to a proportional increase in prepayment intensity; i.e., a one percent decline in the market interest rate would increase the prepayment intensity by $\gamma$ percent. The prepayment due to refinancing is always detrimental to the lender as it will happen when the prevailing market rate is lower than the rate at origination, and thus constitutes a reinvestment risk to the lender.

Remark 4.1 (On the change of measure) Under the physical probability measure, the CIR short rate process has the form (4.1) with parameters $\kappa_{\mathbb{P}}, \theta_{\mathbb{P}}$, and $\sigma$. Taking the market price of interest rate risk in the form $\lambda_{t}=\sigma^{-1} \nu \sqrt{r_{t}}$, the short rate process under the equivalent martingale measure is again CIR (4.1) with parameters $\kappa_{\mathbb{Q}}=\kappa_{\mathbb{P}}+\nu, \theta_{\mathbb{Q}}=\kappa_{\mathbb{P}} \theta_{\mathbb{P}} /\left(\kappa_{\mathbb{P}}+\nu\right)$, and the same volatility $\sigma$. Generally, if the prepayment event (the point process $N_{t}$ ) carries a risk premium, then the $\mathbb{Q}$-intensity could, for some positive constant $\mu$, be equal to $h_{t}^{\mathbb{Q}}=\mu h_{t}^{\mathbb{P}}$. This multiplicative constant is the risk premium to compensate for the prepayment event. However, Jarrow et al. (2005) show that if the event risk (such as defaults by individual companies or prepayments by individual borrowers) can be diversified away, then $\mu=1$ and $h_{t}^{\mathbb{Q}}=h_{t}^{\mathbb{P}}$ (the basic idea of diversifiable jump risk goes back to Merton (1976)). This idea is further discussed by Goncharov (2003) in the context of mortgage prepayments. In the context of our model, if, conditionally on the path of the interest rate process, the prepayment point processes for individual borrowers are independent, the prepayment event risk can be diversified away in a large pool of mortgages, and the functional form of the prepayment intensity as a function of time and the interest rate process is the same under the physical and the risk-neutral measures. Note that this does not imply distributional equivalence since the prepayment intensity is a function of the interest rate process, which changes its drift under the measure change. In this paper we assume that $h_{t}^{\mathbb{Q}}=h_{t}^{\mathbb{P}}$. As our main focus is on pricing, we work with the interest rate process (4.1) under $\mathbb{Q}$ and, to simplify notation, drop the index $\mathbb{Q}$ in $\kappa_{\mathbb{Q}}$ and $\theta_{\mathbb{Q}}$. More generally, if we assume that the prepayment event carries a risk premium and an EMM is not unique, we can simply select an equivalent martingale measure chosen by the market by calibrating the model parameters to the mortgage market data.

Remark 4.2 (On individual mortgage contracts vs. mortgage-backed securities) In this paper we concentrate on the valuation of individual residential mortgage contracts. A mortgage backed security is a share of ownership in a pool with a large number of individual mortgage. Under the homogeneous pool assumption (assuming all mortgages in the pool are the same), the value of the mortgage pool is simply equal to the value of the individual contract times the number of mortgages in the pool. From the standpoint of analyzing the prepayment rate of the mortgage pool (i.e., a fraction of mortgages prepaying per unit time), the prepayment process of the pool is a sum of one-jump point processes of individual mortgages. Since the number of individual mortgages in a pool is very large (typically thousands of mortgages), one can invoke the central limit theorem for processes and show that the prepayment rate of the pool under the homogeneity assumption is equal to the prepayment intensity of the individual mortgage (see Kagraoka (2002) for details of the proof). Furthermore, this can be generalized to non-homogeneous pools (which is typically the case for mortgage-backed securities) by taking an appropriate weighted average.

Remark 4.3 (Prepayment intensity as a function of the short rate vs. the mortgage rate) We specify the prepayment intensity as a function of the short rate. Goncharov (2003), (2004), (2006) and Pliska (2005), (2006) study more general specifications where the prepayment intensity is a function of the currently prevailing mortgage rate $m_{t}=m(t, T)$ rather than the short rate $r_{t}$. This endogenizes the problem. Such models are more realistic, but the 
cost is a significant increase in computational complexity with no hope of analytical tractability and the necessity of intensive use of numerical methods. However, since market interest rates are, in fact, the main factor determining refinancing incentives and mortgage rates, we argue that replacing the currently prevailing mortgage rate with the currently prevailing interest rate as a factor in the prepayment intensity serves as a reasonable first approximation to more general endogenous models, such as the ones developed by Goncharov and Pliska, with the advantage of retaining analytical tractability. Indeed, Longstaff (2005) estimates from market data that mortgage rates and the U.S. Treasury rates are highly correlated, with the correlation coefficient around ninety percent.

Remark 4.4 (On Carr and Linetsky (2000) ESO early exercise model) We remark that our choice of prepayment intensity is somewhat analogous to the early exercise intensity for executive stock options (ESO) proposed by Carr and Linetsky (2000). In that model, the intensity of the ESO early exercise process is assumed to be of the form

$$
h_{t}=\lambda_{f}+\lambda_{e}\left(S_{t}-K\right)^{+}
$$

where $S_{t}$ is the time- $t$ stock prices, $K$ is the stock option strike price, $\lambda_{f}$ is the constant intensity of early exercise due to exogenous reasons (e.g., job termination) and $\lambda_{e}$ is the scale parameter of early exercise due to the executive's desire to obtain liquidity and diversification in the face of increasing stock price as the option goes deeper in the money. In the Carr-Linetsky ESO model the stock price follows geometric Brownian motion, and the authors are able to calculate the Laplace transform of the Brownian area functional in closed form. In the present mortgage prepayment model, the underlying state variable is a CIR interest rate, and the analytical solution is achieved by different means, via the eigenfunction expansion of the relevant FeynmanKac semigroup.

Remark 4.5 (On Commercial Mortgages) We note that the model developed in this paper is for residential mortgages. Commercial mortgages have substantially different characteristics, with the endogenous default decision by commercial borrowers being an important factor in the analysis.

\section{Solution of the Model}

\subsection{Calculating $Q(t, u), H(t, u)$, and $R(t, u)$ by the Eigenfunction Expansion of the Feynman-Kac Semigroup}

The key quantities $Q\left(r_{t} ; t, u\right), H\left(r_{t} ; t, u\right)$, and $R\left(r_{t} ; t, u\right)$ entering the mortgage valuation equations can be interpreted as time- $t$ values of $u$-maturity European-style contingent claims with payoffs $f_{Q}\left(r_{u}\right)=1, f_{H}\left(r_{u}\right)=h_{0}(u)+\gamma\left(k-r_{u}\right)^{+}$, and $f_{R}\left(r_{u}\right)=r_{u}$, respectively, in a fictitious economy with the instantaneous interest rate $r_{s}+h_{0}(s)+\gamma\left(k-r_{s}\right)^{+}$, where the underlying state variable $r_{s}$ follows the CIR diffusion. The problem thus reduces to computing expectations of the form:

$$
\begin{gathered}
\mathbb{E}\left[\exp \left\{-\int_{t}^{u}\left(r_{s}+h_{0}(s)+\gamma\left(k-r_{s}\right)^{+}\right) d s\right\} f\left(r_{u}\right) \mid r_{t}=r\right] \\
=\exp \left\{-\int_{t}^{u} h_{0}(s) d s\right\} \mathbb{E}_{r}\left[\exp \left\{-\int_{0}^{u-t}\left(r_{s}+\gamma\left(k-r_{s}\right)^{+}\right) d s\right\} f\left(r_{u-t}\right)\right],
\end{gathered}
$$


where we took the deterministic factor $e^{-\int_{t}^{u} h_{0}(s) d s}$ outside of the expectation and used the timehomogeneity of the CIR diffusion $\left(\mathbb{E}_{r}[\cdot]=\mathbb{E}\left[\cdot \mid r_{0}=r\right]\right)$. The problem thus further reduces to computing the Feynman-Kac semigroup of the CIR diffusion killed at an additive functional $\int_{0}^{t}\left(r_{s}+\gamma\left(k-r_{s}\right)^{+}\right) d s:$

$$
\mathcal{P}_{t} f(x):=\mathbb{E}_{x}\left[\exp \left\{-\int_{0}^{t}\left(r_{s}+\gamma\left(k-r_{s}\right)^{+}\right) d s\right\} f\left(r_{t}\right)\right] .
$$

In this Section we explicitly construct an eigenfunction expansion of this semigroup. The transition density of the semigroup of the CIR diffusion killed at an additive functional $\int_{0}^{t} r_{s} d s$ is well-known in closed form (Pitman and Yor (1982), Cox et al. (1985); see also Jeanblanc et al. (2006) for a survey). The eigenfunction expansion for this semigroup can be found in Davydov and Linetsky (2003) or Gorovoi and Linetsky (2004) (see also Linetsky (2004a) and (2004b) and Appendix $\mathrm{C}$ for related results). The semigroup of the CIR diffusion killed at an area functional $\int_{0}^{t}\left(r_{s}-a\right)^{+} d s$ was explicitly constructed in Gorovoi and Linetsky (2004). The area functional measures the area below the sample path of the process and above some level $a>0$ (see Perman and Wellner (1996) for the study of Brownian area functionals). Here we are concerned with a more general semigroup of the CIR process killed at an additive functional which is a linear combination of the integral of the process and the area functional $\int_{0}^{t}\left(k-r_{s}\right)^{+} d s$ measuring the area above the sample path of the process and below some level $k>0$. This functional has an appealing intuition in the context of mortgage prepayment modeling, as it plays a role of the hazard process. The longer the interest rate stays below the threshold $k$ and the further it falls below $k$, the larger the value of the hazard process. Fortunately, we are able to generalize the results in Gorovoi and Linetsky (2004) to explicitly compute the eigenfunction expansion of this more general semigroup. A survey of the spectral expansion method for diffusion processes, applications in mathematical finance and further references can be found in Linetsky (2004c).

Recall that the scale and speed densities of the CIR diffusion are (see, e.g., Borodin and Salminen (2002) for a survey of one-dimensional diffusions):

$$
\mathfrak{s}(x)=x^{-\beta} \exp \left(\frac{2 \kappa x}{\sigma^{2}}\right), \mathfrak{m}(x)=\frac{2}{\sigma^{2}} x^{\beta-1} \exp \left(-\frac{2 \kappa x}{\sigma^{2}}\right), \beta:=\frac{2 \kappa \theta}{\sigma^{2}} .
$$

Under Feller's condition $\beta \geq 1$, zero is an inaccessible entrance boundary and $+\infty$ is a nonattracting natural boundary for the CIR process.

Let $\mathcal{H}:=L^{2}((0, \infty), \mathfrak{m})$ be the Hilbert space of real-valued functions square-integrable on $(0, \infty)$ with the speed density $\mathfrak{m}(x)$ and endowed with the inner product

$$
(f, g)=\int_{0}^{\infty} f(x) g(x) \mathfrak{m}(x) d x .
$$

Then Eq. (5.2) defines a contraction semigroup $\left\{\mathcal{P}_{t}, t \geq 0\right\}$ of non-negative symmetric operators in $\mathcal{H}$ (the symmetry refers to the property $\left(\mathcal{P}_{t} f, g\right)=\left(f, \mathcal{P}_{t} g\right)$ and non-negativity to $\left.\left(\mathcal{P}_{t} f, f\right) \geq 0\right)$. Its infinitesimal generator

$$
\mathcal{G} f(x)=\frac{1}{2} \sigma^{2} x f^{\prime \prime}(x)+\kappa(\theta-x) f^{\prime}(x)-\left(x+\gamma(k-x)^{+}\right) f(x)
$$

can be expressed in terms of the scale and speed densities:

$$
\mathcal{G} f(x)=\frac{1}{\mathfrak{m}(x)}\left(\frac{f^{\prime}(x)}{\mathfrak{s}(x)}\right)^{\prime}-\left(x+\gamma(k-x)^{+}\right) f(x) .
$$


Its domain is: $D(\mathcal{G})=\left\{f \in \mathcal{H}: f, f^{\prime} \in A C_{\text {loc }}((0, \infty)), \mathcal{G} f \in \mathcal{H}\right.$, boundary conditions at 0 and $\left.\infty\right\}$, where $A C_{l o c}((0, \infty))$ is the space of functions absolutely continuous over each compact subinterval of $(0, \infty)$, and the appropriate boundary conditions at 0 and $\infty$ are:

$$
\lim _{x \downarrow 0} \frac{f^{\prime}(x)}{\mathfrak{s}(x)}=0, \quad \lim _{x \uparrow \infty} \frac{f^{\prime}(x)}{\mathfrak{s}(x)}=0 .
$$

The infinitesimal generator $\mathcal{G}$ is a self-adjoint and non-positive operator in $\mathcal{H}$. We are interested in its spectral representation, as well as the associated spectral representation of the semigroup of bounded symmetric operators $\left\{\mathcal{P}_{t}, t \geq 0\right\}$.

Theorem 5.1 (i) The operator $\mathcal{G}$ has a purely discrete spectrum with negative eigenvalues $\left\{-\lambda_{n}\right\}_{n=1}^{\infty}$, where $0<\lambda_{1}<\lambda_{2}<\ldots$, and the corresponding continuous eigenfunctions $\left\{\varphi_{n}(x)\right\}_{n=1}^{\infty}$ with continuous first derivatives and normalized so that $\left(\varphi_{n}, \varphi_{m}\right)=\delta_{n, m}\left(\delta_{n, m}=1(0)\right.$ when $n=m(n \neq m)$ is the Kroeneker symbol).

(ii) Let $\lambda \in(0, \infty)$. Introduce the following notation ( $\beta$ was defined in Eq.(5.3)):

$$
\begin{gathered}
\rho_{1}:=\sqrt{2(1-\gamma) \sigma^{2}+\kappa^{2}}, \rho_{2}:=\sqrt{2 \sigma^{2}+\kappa^{2}}, \alpha_{1}:=\frac{2 \rho_{1}}{\sigma^{2}}, \alpha_{2}:=\frac{2 \rho_{2}}{\sigma^{2}}, \\
a_{1}:=\frac{\beta}{2}-\frac{\kappa^{2} \theta}{\sigma^{2} \rho_{1}}, a_{2}:=\frac{\beta}{2}-\frac{\kappa^{2} \theta}{\sigma^{2} \rho_{2}}, \\
\psi_{\lambda}(x):=\exp \left(\frac{\left(\kappa-\rho_{1}\right) x}{\sigma^{2}}\right) M\left(a_{1}-(\lambda-\gamma k) / \rho_{1}, \beta, \alpha_{1} x\right), x \in(0, k], \\
\phi_{\lambda}(x):=\exp \left(\frac{\left(\kappa-\rho_{2}\right) x}{\sigma^{2}}\right) U\left(a_{2}-\lambda / \rho_{2}, \beta, \alpha_{2} x\right), x \in[k,+\infty),
\end{gathered}
$$

where $M(a, b, z)$ and $U(a, b, z)$ are the Kummer and Tricomi confluent hypergeometric functions. $^{2}$ Define a function of $\lambda$ (the scale density-normalized Wronskian of the functions $\psi_{\lambda}(x)$ and $\phi_{\lambda}(x)$ evaluated at the point $x=k$; the prime denotes differentiation in $\left.x\right)$ :

$$
\begin{gathered}
w(\lambda):=\frac{1}{s(k)}\left(\psi_{\lambda}(k) \phi_{\lambda}^{\prime}(k)-\psi_{\lambda}^{\prime}(k) \phi_{\lambda}(k)\right) \\
=k^{\beta} e^{-\left(\rho_{1}+\rho_{2}\right) k / \sigma^{2}}\left\{\sigma^{-2}\left(\rho_{1}-\rho_{2}\right) M\left(a_{1}-(\lambda-\gamma k) / \rho_{1}, \beta, \alpha_{1} x\right) U\left(a_{2}-\lambda / \rho_{2}, \beta, \alpha_{2} x\right)\right. \\
-\alpha_{2}\left(a_{2}-\lambda / \rho_{2}\right) M\left(a_{1}-(\lambda-\gamma k) / \rho_{1}, \beta, \alpha_{1} x\right) U\left(a_{2}-\lambda / \rho_{2}+1, \beta+1, \alpha_{2} x\right) \\
\left.-\beta^{-1} \alpha_{1}\left(a_{1}-(\lambda-\gamma k) / \rho_{1}\right) M\left(a_{1}-(\lambda-\gamma k) / \rho_{1}+1, \beta+1, \alpha_{1} x\right) U\left(a_{2}-\lambda / \rho_{2}, \beta, \alpha_{2} x\right)\right\} .
\end{gathered}
$$

Then the negatives of the eigenvalues of the infinitesimal generator $\mathcal{G}, 0<\lambda_{1}<\lambda_{2}<\ldots$, can be identified with positive simple zeros of the Wronskian $w(\lambda)$, i.e., roots of the equation

$$
w(\lambda)=0
$$

\footnotetext{
${ }^{2}$ See Appendix B and Abramowitz and Stegun (1972) and Slater (1960) for their properties. These functions are available as built-in functions in the Mathematica software system with the calls Hypergeometric1F1 and HypergeometricU.
} 
on the positive half-line, and the corresponding normalized eigenfunctions $\left\{\varphi_{n}(x)\right\}_{n=1}^{\infty}$ can be taken in the form:

$$
\varphi_{n}(x)=\left\{\begin{array}{ll}
\sqrt{\frac{\phi_{\lambda_{n}}(k)}{\Delta_{n} \psi_{\lambda_{n}}(k)}} \psi_{\lambda_{n}}(x), & x \in(0, k] \\
\sqrt{\frac{\psi_{\lambda_{n}}(k)}{\Delta_{n} \phi_{\lambda_{n}}(k)}} \phi_{\lambda_{n}}(x), & x \in[k, \infty)
\end{array},\right.
$$

where

$$
\Delta_{n}:=\left.\frac{d w(\lambda)}{d \lambda}\right|_{\lambda=\lambda_{n}}
$$

Proof. See Appendix A.

The infinitesimal generator $\mathcal{G}$ and the semigroup operators $\mathcal{P}_{t}$ with $t>0$ share the same eigenfunctions $\left\{\varphi_{n}\right\}$ that form an orthonormal basis in $\mathcal{H}$. If $-\lambda_{n}$ are the eigenvalues of $\mathcal{G}$, then $e^{-\lambda_{n} t}$ are the eigenvalues of $\mathcal{P}_{t}$, and we have the following eigenfunction expansion for conditional expectations (5.2) with $f \in \mathcal{H}$.

Corollary 5.1 For any $f \in \mathcal{H}$ and $t>0$, the function $\mathcal{P}_{t} f(x)$ has the following eigenfunction expansion:

$$
\mathcal{P}_{t} f(x)=\sum_{n=1}^{\infty} e^{-\lambda_{n} t} c_{n}^{f} \varphi_{n}(x),
$$

where the expansion coefficients are

$$
c_{n}^{f}=\left(f, \varphi_{n}\right) .
$$

Theorem 5.1 and Corollary 5.1 provide a recipe to calculate expectations (5.2) for $f \in \mathcal{H}$. First, the eigenvalues are determined by finding the roots of the Wronskian $\lambda_{n}$ by numerically solving Eq.(5.12) (we used the Mathematica FindRoot routine to do that). Then the corresponding eigenfunctions are given by Eq.(5.13) in terms of the confluent hypergeometric functions (the derivative of the Wronskian evaluated at the eigenvalue in Eq.(5.14) is calculated by using the derivatives of the confluent hypergeometric functions with respect to their first index given in Appendix B), and the expansion coefficients (5.16) are calculated by integrating $f(x) \varphi_{n}(x)$ with respect to the speed measure $\mathfrak{m}(x) d x$ to compute the inner product (5.4) (we used the Mathematica numerical integration routine NIntegrate for this computation). Finally, the result for the expectation (5.2) is given by the eigenfunction expansion (5.15). For this semigroup, $\lambda_{n}$ increase approximately linearly with $n$, and the higher order terms are suppressed with the factors $e^{-\lambda_{n} t}$, helping convergence of the eigenfunction expansion (note that the expansion (5.15) converges in $\mathcal{H}$ even for $t=0$, in which case it is the $L^{2}$-expansion of the function $f \in \mathcal{H}$ in the eigenfunction basis $\left\{\varphi_{n}\right\}$; for $t>0$ the convergence is accelerated as the factors $e^{-\lambda_{n} t}$ help convergence by suppressing the higher order terms).

The quantities $Q(r ; t, u)$ and $R(r ; t, u)$ have the form

$$
e^{-\int_{t}^{u} h_{0}(s) d s} \mathcal{P}_{u-t} f(r)=e^{-\int_{t}^{u} h_{0}(s) d s} \sum_{n=1}^{\infty} e^{-\lambda_{n}(u-t)} c_{n}^{f} \varphi_{n}(r)
$$

with $f_{Q}(r)=1$ and $f_{R}(r)=r$, respectively. The quantity $H\left(r_{t} ; t, u\right)$ has the form

$$
H\left(r_{t} ; t, u\right)=e^{-\int_{t}^{u} h_{0}(s) d s}\left[h_{0}(u) Q\left(r_{t} ; t, u\right)+\gamma A\left(r_{t} ; t, u\right)\right],
$$


where

$$
A(r ; t, u)=\mathcal{P}_{u-t} f_{A}(r)
$$

with $f_{A}(r)=(k-r)^{+}$. All three functions $f_{Q}, f_{A}$, and $f_{R}$ are in $\mathcal{H}$ (square-integrable with the speed density (5.3)) and, hence, the corresponding expectations $\mathcal{P}_{u-t} f(r)$ can be calculated by the eigenfunction expansion method. In what follows we denote the corresponding expansion coefficients by $c_{n}^{Q}, c_{n}^{A}$, and $c_{n}^{R}$, respectively. The three quantities $Q, R$, and $H$ are also related by Eq.(3.8).

\subsection{Calculating Time Integrals}

\subsubsection{Continuous Payments}

To compute the value of the mortgage (3.6), the interest-only (IO) part (3.11), the principal-only (PO) part (3.12), and determine the mortgage rate $m$ by solving Eq.(3.9), we need to calculate the integrals with respect to time entering those expressions:

$$
\int_{t}^{T}\left(1-e^{-m(T-u)}\right) e^{-\int_{t}^{u} h_{0}(s) d s} \mathcal{P}_{u-t} f(r) d u=\sum_{n=1}^{\infty} L_{c}\left(t, T ; \lambda_{n}, m\right) c_{n}^{f} \varphi_{n}(r),
$$

where

$$
L_{c}\left(t, T ; \lambda_{n}, m\right)=\int_{t}^{T}\left(1-e^{-m(T-u)}\right) e^{-\int_{t}^{u} h_{0}(s) d s-\lambda_{n}(u-t)} d u .
$$

Fortunately, we are able to calculate these integrals in closed form for both the CPR and PSA specifications of $h_{0}(t)$. For the CPR specification $h_{0}=$ const, we obtain:

$$
L_{c, C P R}\left(t, T ; \lambda_{n}, m\right)=\frac{m\left(1-e^{-\lambda_{n}^{*}(T-t)}\right)-\lambda_{n}^{*}\left(1-e^{-m(T-t)}\right)}{\lambda_{n}^{*}\left(m-\lambda_{n}^{*}\right)}, \text { where } \lambda_{n}^{*}=h_{0}+\lambda_{n} .
$$

For the PSA specification (4.3), if $t \geq T^{*}$, the integral is the same as for the CPR with $h_{0}=a b T^{*}$ since $h_{0}(t)$ is constant and equal to $h_{0}=a b T^{*}$ for $t \geq T^{*}$. For $0 \leq t<T^{*}$, we have

$$
\int_{t}^{u} h_{0}(s) d s=\frac{a b}{2}\left(\left(u \wedge T^{*}\right)^{2}-t^{2}\right)+\mathbf{1}_{\left\{u>T^{*}\right\}} a b T^{*}\left(u-T^{*}\right)
$$

and we need to consider two cases. When $T \leq T^{*}$, we obtain

$$
\begin{gathered}
L_{c, P S A}\left(t, T ; \lambda_{n}, m\right)=\int_{t}^{T}\left(1-e^{-m(T-u)}\right) e^{-a b\left(u^{2}-t^{2}\right) / 2-\lambda_{n}(u-t)} d u \\
=\sqrt{\frac{\pi}{2 a b}} \exp \left(\frac{\left(a b t+\lambda_{n}\right)^{2}}{2 a b}\right)\left\{\operatorname{Erf}\left(\frac{\lambda_{n}+a b T}{\sqrt{2 a b}}\right)-\operatorname{Erf}\left(\frac{\lambda_{n}+a b t}{\sqrt{2 a b}}\right)\right. \\
\left.+\exp \left(\frac{m\left(m-2\left(a b T+\lambda_{n}\right)\right)}{2 a b}\right)\left(\operatorname{Erf}\left(\frac{\lambda_{n}-m+a b T}{\sqrt{2 a b}}\right)-\operatorname{Erf}\left(\frac{\lambda_{n}-m+a b t}{\sqrt{2 a b}}\right)\right)\right\},
\end{gathered}
$$

where $\operatorname{Erf}(x)$ is the error function, $\operatorname{Erf}(x)=\frac{2}{\sqrt{\pi}} \int_{0}^{x} e^{-y^{2}} d y$. When $T>T^{*}$, we obtain

$$
L_{c, P S A}\left(t, T ; \lambda_{n}, m\right)=\int_{t}^{T^{*}}\left(1-e^{-m(T-u)}\right) e^{-a b\left(u^{2}-t^{2}\right) / 2-\lambda_{n}(u-t)} d u
$$




$$
\begin{gathered}
+\int_{T^{*}}^{T}\left(1-e^{-m(T-u)}\right) e^{-a b\left(\left(T^{*}\right)^{2}-t^{2}\right) / 2-a b T^{*}\left(u-T^{*}\right)-\lambda_{n}(u-t)} d u \\
=\sqrt{\frac{\pi}{2 a b}} \exp \left(\frac{\left(a b t+\lambda_{n}\right)^{2}}{2 a b}\right)\left\{\operatorname{Erf}\left(\frac{a b T^{*}+\lambda_{n}}{\sqrt{2 a b}}\right)-\operatorname{Erf}\left(\frac{a b t+\lambda_{n}}{\sqrt{2 a b}}\right)\right. \\
\left.+\exp \left(\frac{m\left(m-2\left(a b T+\lambda_{n}\right)\right)}{2 a b}\right)\left(\operatorname{Erf}\left(\frac{\lambda_{n}-m+a b T^{*}}{\sqrt{2 a b}}\right)-\operatorname{Erf}\left(\frac{\lambda_{n}-m+a b t}{\sqrt{2 a b}}\right)\right)\right\} \\
+\frac{1}{\left(a b T^{*}+\lambda_{n}-m\right)\left(a b T^{*}+\lambda_{n}\right)}\left\{m \exp \left(-\frac{1}{2} a b\left(T^{*}\left(2 T-T^{*}\right)-t^{2}\right)-\lambda_{n}(T-t)\right)\right. \\
\left.+\exp \left(-\frac{a b}{2}\left(\left(T^{*}\right)^{2}-t^{2}\right)-\lambda_{n}\left(T^{*}-t\right)\right)\left(\left(a b T^{*}+\lambda_{n}\right)\left(1-e^{-m\left(T-T^{*}\right)}\right)-m\right)\right\} .
\end{gathered}
$$

\subsubsection{Discrete Payments}

To compute the value of the mortgage (3.15), IO (3.16), PO (3.17), and determine the mortgage rate $m$ by solving Eq.(3.18), we need to calculate the integral with respect to time entering those expressions. For the CPR specification we have for $t=0\left(\right.$ where $\left.\lambda_{n}^{*}=h_{0}+\lambda_{n}\right)$ :

$$
\begin{gathered}
L_{d, C P R}\left(0, T ; \lambda_{n}, m\right)=\int_{0}^{T}(1+m(u-[u / \Delta] \Delta))\left(1-(1+m \Delta)^{[u / \Delta]-N}\right) e^{-\lambda_{n}^{*} u} d u \\
=\sum_{k=1}^{N} \int_{(k-1) \Delta}^{k \Delta}(1+m(u-(k-1) \Delta))\left(1-(1+m \Delta)^{k-1-N}\right) e^{-\lambda_{n}^{*} u} d u \\
=\frac{\left(m+\lambda_{n}^{*}\right)\left(e^{\Delta \lambda_{n}^{*}}-1\right)-m \lambda_{n}^{*} \Delta}{\left(\lambda_{n}^{*}\right)^{2}} \sum_{k=1}^{N} e^{-k \Delta \lambda_{n}^{*}}\left(1-(1+m \Delta)^{k-1-N}\right) \\
=\frac{\left(\left(m+\lambda_{n}^{*}\right)\left(e^{\Delta \lambda_{n}^{*}}-1\right)-m \lambda_{n}^{*} \Delta\right)\left(\left(e^{\Delta \lambda_{n}^{*}}-1\right)\left(1-(1+m \Delta)^{-N}\right)-m \Delta\left(1-e^{-\lambda_{n}^{*} T}\right)\right)}{\left(\lambda_{n}^{*}\right)^{2}\left(e^{\left.\Delta \lambda_{n}^{*}-1\right)\left(e^{\Delta \lambda_{n}^{*}}-1-m \Delta\right)} .\right.}
\end{gathered}
$$

To compute this integral, we first broke it up into the sum of integrals over the subintervals $[(k-1) \Delta, k \Delta)$, calculated each integral, and then calculated the sum in closed form. It is easy to verify that when $N \rightarrow \infty$, Eq.(5.23) converges to the continuous case (5.20). For $0<t<T$, we have (where $\lambda_{n}^{*}=h_{0}+\lambda_{n}$ ):

$$
\begin{gathered}
L_{d, C P R}\left(t, T ; \lambda_{n}, m\right)=\int_{t}^{T}(1+m(u-[u / \Delta] \Delta))\left(1-(1+m \Delta)^{[u / \Delta]-N}\right) e^{-\lambda_{n}^{*} u} d u \\
=\int_{t}^{[t / \Delta] \Delta}(1+m(u-([t / \Delta]-1) \Delta))\left(1-(1+m \Delta)^{[t / \Delta]-1-N}\right) e^{-\lambda_{n}^{*}(u-t)} d u \\
+\sum_{k=[t / \Delta]+1}^{N} \int_{(k-1) \Delta}^{k \Delta}(1+m(u-(k-1) \Delta))\left(1-(1+m \Delta)^{k-1-N}\right) e^{-\lambda_{n}^{*}(u-t)} d u \\
=\frac{1}{\left(\lambda_{n}^{*}\right)^{2}}\left(1-(1+m \Delta)^{[t / \Delta]-1-N}\right) \\
\quad \times\left(m+\lambda_{n}^{*}+m \lambda_{n}^{*}(t-([t / \Delta]-1) \Delta)-e^{-\lambda_{n}^{*}([t / \Delta] \Delta-t)}\left(m+\lambda_{n}^{*}+m \lambda_{n}^{*} \Delta\right)\right)
\end{gathered}
$$




$$
+e^{\lambda_{n}^{*} t} L_{d, C P R}\left(0, T ; \lambda_{n}, m\right)-e^{\lambda_{n}^{*} t} L_{d, C P R}\left(0,[t / \Delta] \Delta ; \lambda_{n}, m\right)
$$

For the PSA specification with discrete payments, unfortunately the sum cannot be calculated in closed form and the resulting expression is computationally less efficient than the continuous PSA case. For the 30-year mortgage, the expression for $L_{d, P S A}\left(0, T ; \lambda_{n}, m\right)$ is a sum of 360 terms, each involving error functions, in contrast to a single term in the continuous PSA case, as well as the discrete CPR case. Since we also need to compute the outer sum in $n$ to evaluate the eigenfunction expansion, this becomes computationally expensive. Fortunately, as we demonstrate in Section 6, the continuous payments case provides an excellent approximation for the discrete payments case (the mortgage rates $m$ determined under the continuous and discrete specifications differ by less than one basis point), and for all practical purposes the continuous PSA specification provides and excellent approximation for the discrete PSA specification.

\subsection{Solving the Non-Linear Equation for the Mortgage Rate}

To determine the mortgage rate $m=m(0, T)$ for the mortgage with continuous payments we need to solve the non-linear equation (3.9). Substituting the eigenfunction expansions for $R$ and $Q$ into the equation, using the results of the previous section for the integrals with respect to time, and rearranging terms, we arrive at the following non-linear equation for $m$ :

$$
\sum_{n=1}^{\infty} \varphi_{n}(r)\left(m c_{n}^{Q}-c_{n}^{R}\right) L_{c}\left(0, T ; \lambda_{n}, m\right)=0 .
$$

Since analytical expressions for the derivative of the left-hand side with respect to $m$ are available under both the CPR and PSA specifications, we solve this non-linear equation numerically by the Newton-Raphson iterations (we first truncate the infinite summation at an appropriate finite level and then implement Newton-Raphson iterations). The natural starting point is to choose the mortgage rate equal to the initial short rate, i.e., $m_{0}=r$. In our numerical experiments the Newton-Raphson algorithm converged to the accuracy of one basis point for the mortgage rate after just two iterations.

For the mortgage with discrete payments under the CPR specification we need to solve the non-linear equation (3.18). Using Eq.(5.17) and the results of the previous Section, calculating the sum in $k$ in (3.18) in closed form and rearranging terms, we arrive at the following non-linear equation for the mortgage rate $m$ :

$$
\sum_{n=1}^{\infty} \varphi_{n}(r)\left\{m \Delta c_{n}^{Q}\left(\frac{1-e^{-\lambda_{n}^{*} T}}{e^{\lambda_{n}^{*} \Delta}-1}\right)+\left(h_{0} c_{n}^{Q}+\gamma c_{n}^{A}\right) L_{d, C P R}\left(0, T ; \lambda_{n}, m\right)\right\}=1-(1+m \Delta)^{-N} .
$$

Since analytical expressions for the derivative of the left-hand side with respect to $m$ are available, we solve this non-linear equation numerically by the Newton-Raphson iterations starting at $m_{0}=r$. In our numerical experiments the Newton-Raphson algorithm converged to the accuracy of one basis point for the mortgage rate after just two iterations, just as in the continuous case. 


\subsection{Valuing Seasoned Mortgages and Calculating Mortgage Delta}

The time- $t$ value of a seasoned mortgage with continuous payments originated at time zero is given by Eq.(3.6). Using the results of the previous sections, it reduces to

$$
M_{t}=P_{t}\left\{1+\sum_{n=1}^{\infty} \varphi_{n}(r)\left(m c_{n}^{Q}-c_{n}^{R}\right) \frac{L_{c}\left(t, T ; \lambda_{n}, m\right)}{1-e^{-m(T-t)}}\right\} .
$$

The interest-only part given by Eq.(3.11) reduces to

$$
I O_{t}=m P_{t} \sum_{n=1}^{\infty} \varphi_{n}(r) c_{n}^{Q} \frac{L_{c}\left(t, T ; \lambda_{n}, m\right)}{1-e^{-m(T-t)}} .
$$

The time- $t$ value of a seasoned mortgage with discrete payments and CPR specification Eq.(3.15) reduces to

$$
\begin{gathered}
M_{t}=\frac{P_{0}}{1-(1+m \Delta)^{-N}} \\
\times \sum_{n=1}^{\infty} \varphi_{n}(r)\left\{m \Delta c_{n}^{Q}\left(\frac{e^{-\lambda_{n}^{*}([t / \Delta] \Delta-t)}-e^{-\lambda_{n}^{*}(T-t)}}{e^{\lambda_{n}^{*} \Delta}-1}\right)+\left(h_{0} c_{n}^{Q}+\gamma c_{n}^{A}\right) L_{d, C P R}\left(t, T ; \lambda_{n}, m\right)\right\} .
\end{gathered}
$$

One of the advantages of the eigenfunction expansion approach is that the mortgage delta, the first derivative of the mortgage value function with respect to the interest rate $r$ measuring the sensitivity of the mortgage value to changes in interest rates, can be obtained by differentiating the eigenfunction expansion with respect to $r$. Derivatives of the eigenfunctions $d \varphi_{n}(r) / d r$ are obtained using the derivatives of the confluent hypergeometric functions given in Appendix B. Since the eigenvalues $\lambda_{n}$ and expansion coefficients $c_{n}$ are the same as in the expansion for the value function, the mortgage delta is obtained with essentially no additional computational effort.

\section{Implementation and Case Studies}

\subsection{Computational Implementation}

In order to illustrate computational performance of the eigenfunction expansion method, we fix the following parameters for the CIR process: $\theta=0.06, \kappa=0.25$, and $\sigma=0.1$, and consider mortgage contracts with continuous payments and prepayment intensity (4.2) with parameters $h_{0}=0.045, \gamma=5$ and $k=r_{0}$ (CPR specification for the exogenous prepayment component). Table 1 presents the first ten eigenvalues $\lambda_{n}$ and expansion coefficients $c_{n}^{Q}$ and $c_{n}^{R}$. We observe that the eigenvalues increase approximately linearly with $n$ and the expansion coefficients decrease rapidly with $n$. To determine the mortgage rate $m=m(0, T)$ for a $T$ maturity mortgage, we truncate the expansions in the non-linear equation (5.25) for the mortgage rate at some level $K$ and then perform Newton-Raphson iterations starting from $m_{0}=r_{0}$. In this example we assume $r_{0}=0.09$. Table 2 shows convergence of the eigenfunction expansion to determine $m$ for a range of maturities $T$. In the left column the truncation level $K$ is indicated (i.e., the terms with $n=1, \ldots, K$ are included in the expansion and the remaining terms with $K+1, K+2, \ldots$ are discarded). The table entries give the corresponding mortgage rates determined to the accuracy of one basis point. We see that summing up the first five 


\begin{tabular}{||c||ccc||}
\hline$n$ & $\lambda_{n}$ & $c_{n}^{R}$ & $c_{n}^{Q}$ \\
\hline 1 & 0.20734 & 0.003803 & 0.054054 \\
2 & 0.47884 & $-9.2333 \times 10^{-4}$ & 0.015381 \\
3 & 0.74412 & $1.2171 \times 10^{-4}$ & 0.006317 \\
4 & 1.02106 & $-1.3772 \times 10^{-4}$ & 0.001281 \\
5 & 1.29713 & $-7.8077 \times 10^{-5}$ & $-1.0992 \times 10^{-4}$ \\
6 & 1.57718 & $-3.4895 \times 10^{-5}$ & $-2.6412 \times 10^{-4}$ \\
7 & 1.85936 & $-3.6717 \times 10^{-6}$ & $-9.6048 \times 10^{-5}$ \\
8 & 2.14140 & $9.3305 \times 10^{-6}$ & $4.6027 \times 10^{-5}$ \\
9 & 2.42418 & $1.0297 \times 10^{-6}$ & $9.2959 \times 10^{-5}$ \\
10 & 2.70820 & $5.9369 \times 10^{-6}$ & $7.2403 \times 10^{-5}$ \\
\hline
\end{tabular}

Table 1: Eigenvalues and expansion coefficients. Model parameters: $\theta=0.06, \kappa=0.25, \sigma=0.1$, $h_{0}=0.045, \gamma=5, r_{0}=0.09$, and $k=r_{0}$.

terms in the eigenfunction expansion produces the result for the mortgage rate to the accuracy of one basis point. Table 3 shows convergence of the Newton-Raphson iterations for the same range of maturities and parameters. The iterations are started at $m_{0}=r_{0}$ and $m_{i}, i=1,2,3,4$ are the values of the mortgage rate on the $i$ th iteration. We observe that the Newton-Raphson iterations converge to the accuracy of one basis point in just two iterations. Computational performance in solving the non-linear equation (5.26) for mortgage rates with discrete payments is very similar.

Table 4 gives mortgage rates determined under the continuous and monthly $(\Delta=1 / 12)$ payments assumption, both with and without prepayments. We see that for the contracts without prepayments the differences are about 2.24 basis points for one year maturity and decline for longer maturities. For the contracts with prepayments the differences are about 0.8 basis points for one year maturity and decline to a negligible 0.002 basis points for thirty-year mortgages. The reason why the differences between the contracts with continuous and discrete payments are lower with prepayments is because we allow continuous prepayment. That is, even though scheduled interest and principal payments happen discretely (monthly) in the contract with discrete payments, the mortgagor is allowed to prepay at any time (not necessarily at the end of the month), as is done in practice (the mortgage prepayment typically can occur on any day of the month, with the interest accruing up to the date of prepayment). We can thus conveniently approximate mortgage contracts with discrete payments with the contracts with continuous payments. While under the CPR assumption of constant $h_{0}$ both discrete and continuous cases exhibit similar computational performance, under the time-dependent PSA assumption continuous case is more computationally efficient as the function $L_{c, P S A}$ is available in closed form as discussed in section 5.2. Therefore, we will assume continuous payments when working with the PSA specification in the next section.

\subsection{Case Studies}

\subsubsection{Mortgage Rates}

We now investigate the effects of parameters on the mortgage rates. The parameters of the CIR process are the same as in the previous Section, and we consider mortgages with continuous 


\begin{tabular}{||c||cccccc||}
\hline$K$ & $T=1$ & 2 & 5 & 10 & 20 & 30 \\
\hline 3 & 0.0884 & 0.0866 & 0.0832 & 0.0807 & 0.0791 & 0.0787 \\
5 & 0.0879 & 0.0862 & 0.0829 & 0.0805 & 0.0790 & 0.0786 \\
10 & 0.0878 & 0.0861 & 0.0829 & 0.0805 & 0.0790 & 0.0785 \\
15 & 0.0878 & 0.0861 & 0.0829 & 0.0805 & 0.0790 & 0.0785 \\
\hline
\end{tabular}

Table 2: Convergence of the eigenfunction expansion in determining the mortgage rates. The table presents mortgage rates computed by truncating the eigenfunction expansion after $K$ terms. Model parameters: $\theta=0.06, \kappa=0.25, \sigma=0.1, h_{0}=0.045, \gamma=5, r_{0}=0.09$, and $k=r_{0}$.

\begin{tabular}{||c||cccccc||}
\hline$i$ & $T=1$ & 2 & 5 & 10 & 20 & 30 \\
\hline 1 & 0.08784 & 0.08624 & 0.08332 & 0.08112 & 0.07952 & 0.07888 \\
2 & 0.08780 & 0.08609 & 0.08287 & 0.08047 & 0.07895 & 0.07853 \\
3 & 0.08780 & 0.08609 & 0.08287 & 0.08047 & 0.07895 & 0.07853 \\
Exact & 0.087796 & 0.086090 & 0.082865 & 0.080465 & 0.078952 & 0.078527 \\
\hline
\end{tabular}

Table 3: Convergence of the Newton-Raphson iterations in determining the mortgage rates. Model parameters: $\theta=0.06, \kappa=0.25, \sigma=0.1, h_{0}=0.045, \gamma=5, r_{0}=0.09$, and $k=r_{0}$.

\begin{tabular}{||c||cccc||}
\hline & $T=1$ & 5 & 15 & 30 \\
\hline Continuous no prepayment & 0.0876023 & 0.0800277 & 0.0705366 & 0.0656842 \\
Discrete no prepayment & 0.0878263 & 0.0802309 & 0.0707208 & 0.0658566 \\
Difference (bp) & 2.240 & 2.032 & 1.842 & 1.725 \\
\hline Continuous with prepayment & 0.0877957 & 0.0828653 & 0.0794494 & 0.0785272 \\
Discrete with prepayment & 0.0877149 & 0.0828342 & 0.0794448 & 0.0785270 \\
Difference (bp) & 0.808 & 0.311 & 0.047 & 0.002 \\
\hline
\end{tabular}

Table 4: Comparison of mortgage rates obtained under discrete (monthly) and continuous payment assumptions. Model parameters: $\theta=0.06, \kappa=0.25, \sigma=0.1, r_{0}=0.09$. The cases without prepayment and with prepayment with parameters $h_{0}=0.045, \gamma=5$, and $k=r_{0}$ are presented. 
payments and prepayment intensity (4.2) with the PSA specification (4.3) for $h_{0}(t)$. We thus have two prepayment parameters $b$ and $\gamma$. The interest rate threshold is set to $k=r_{0}-s$ with $s=0.0025$ (a spread of 25 basis points is subtracted to account for refinancing transaction costs). Figure 1 plots the term structure of mortgage rates for three different starting interest rate levels: $r_{0}=0.03, r_{0}=0.06, r_{0}=0.09$. On each of the three graphs, we plot four term structures of mortgage rates: without prepayment, with exogenous prepayment only $(b=0.75$, or $75 \%$ of PSA, and $\gamma=0$ ), interest rate-driven prepayment due to refinancing only with $\gamma=10$ and $b=0$, and both exogenous and interest rate-driven refinancing prepayments $(b=0.75$, $\gamma=10)$.

When $r_{0}=0.03$, the current rate is much lower than the long-run level $\theta=0.06$ and, hence, the rates are expected to increase in the future towards the long-run level due to mean reversion. As a result, the term structure of mortgage rates without prepayment is steeply upward-sloping. Compared to the mortgage rates without prepayment, the mortgage rates with the exogenous prepayment only $(b=0.75, \gamma=0)$ are somewhat lower than the rates without prepayment (the prepayment spread is negative in this case). This makes intuitive sense: interest rates will tend to increase, but the mortgagors will still prepay at the fixed exogenous rate, which is clearly sub-optimal as they will be prepaying during the times with higher interest rates. As expected, the mortgage rates with the interest rate-driven refinancing only $(b=0, \gamma=10)$ are higher than the rates without prepayment, and the prepayment spread is positive but small, at 19 basis points for the 30-year mortgage. The model with $b=0$ and $\gamma>0$ always exhibits a strictly positive prepayment spread (as the interest rate-driven refinancing is the only reason for prepayment in this model, and this is always detrimental for the lender, as the lender will have to re-lend at lower rates), but in this case the spread is relatively small since the probability of the rate to decline further from the current low level is small due to the mean-reverting nature of the interest rate process. Finally, the case with $b=0.75$ and $\gamma=10$ exhibits prepayment spreads very close to zero, as the positive prepayment spread arising from the interest rate-driven refinancing (due to $\gamma>0$ ) is effectively canceled with the negative prepayment spread arising from the sub-optimal exogenous prepayment (due to $b>0$ ).

Moving on to the case of high current interest rate $r_{0}=0.09$ (significantly greater than the long-run level $\theta=0.06$ ), we observe the opposite behavior. The term structure of mortgage rates without prepayment is downward-sloping, as the rates are expected to decline towards the long-run mean due to mean reversion. Prepayment spreads generated by the model with exogenous prepayments only $(b=0.75, \gamma=0)$ are moderately positive but not very large. However, prepayment spreads generated by the model with the interest rate-driven refinancing only $(b=0, \gamma>0)$ are very large, reaching as much as 170 basis points for the 30-year mortgage in this example. Again, this makes intuitive sense as the rates are expected to decline substantially from the current high levels, and the mortgage will likely be prepaid and the lender will have to re-lend at the lower rates prevailing in the future. Hence, the lender requires some compensation for this reinvestment risk in the form of the substantial prepayment spread above the mortgage rates that would be quoted on a contract without the possibility of prepayment. Finally, adding exogenous prepayment to the interest rate-driven one (both $b>0$ and $\gamma>0$ ) does not add to the spread substantially, as the mortgage will likely be prepaid due to declining interest rates anyway.

The intermediate case with $r_{0}=\theta=0.06$ is in the middle between the two extremes of behavior. The term structure of mortgage rates without prepayment is mildly downward sloping due to convexity effects. Adding exogenous prepayment adds a very narrow prepayment spread 
to the mortgage rate. Given the current rate is equal to the long-run level, future rate increases and declines are roughly equally likely, and, hence, it is roughly equally likely that exogenous prepayment would happen at lower or at higher rates and be either detrimental or positive for the lender. We thus do not expect a significant prepayment spread either way from adding exogenous prepayment. Adding prepayment due to the interest-rate driven refinancing does add a substantial positive prepayment spread. Indeed, the spread is 66 basis points for the 30-year mortgage in this example. It is substantially larger than the prepayment spread for the low rate regime with $r_{0}=0.03$, but substantially smaller than the spread for the high rate regime with $r_{0}=0.09$. Finally, adding exogenous prepayment on top of the interest rate-driven refinancing does not alter the prepayment spread appreciably.

\subsubsection{Seasoned Mortgages}

We now turn to the problem of valuing seasoned mortgages. Suppose initially the short rate is $r_{0}=0.06(6 \%)$. The CIR model parameters are the same as in the previous example. Again, four cases are considered: mortgage with no prepayment, exogenous prepayment $(b>0, \gamma=0)$, interest rate-driven refinancing $(b=0, \gamma>0)$, and the combination of both $(b>0, \gamma>0)$. Mortgage rates for a 30-year mortgage in these four cases are: $5.74 \%, 5.77 \%, 6.40 \%$, and $6.36 \%$ (note that in this scenario adding exogenous prepayment to refinancing actually lowers the mortgage rate, as the mortgagor may end up prepaying suboptimally while the rates are still high due to exogenous prepayment instead of waiting to refinance at lower rate). Figure 2 plots value functions $M(r ; t, T)$ of these four 30-year mortgages with $P_{0}=\$ 100$ as functions of the current interest rate $r(t=0, T=30)$. It also plots their IO and PO parts, as well as their deltas.

When the short rate is $6 \%$ all four mortgages are worth par by construction. We now analyze how the mortgage value will react to changes in market interest rates. The mortgage without prepayment is just an annuity, its value is a decreasing function of the short rate and its delta is negative. Adding interest-rate driven refinancing decreases the sensitivity to changes in the rate, especially for lower rates. When the rates decline, the value of the mortgage annuity increases, but since the mortgagor will tend to prepay when the rates decline, increase in the value of the mortgage with prepayment is significantly smaller than increase in the value of the comparable mortgage without prepayment (the mortgage with prepayment is significantly less valuable to the lender when rates decline), and the absolute value of the mortgage delta is significantly smaller for low rates. As rates increase, the value of the mortgage with prepayment declines less than the value of the comparable mortgage without prepayment. This is due to the higher mortgage rate on the mortgage with refinancing ( $6.40 \%$ vs. $5.74 \%$ in this example). When rates increase, the prepayment option granted to the mortgagor by the lender is less valuable and the mortgage with prepayment behaves more like a comparable mortgage without prepayment (as there will be no prepayments at high rates). But since the mortgage rate is higher on the mortgage with prepayment, it will tend to hold its value better as the rates increase. Adding exogenous prepayment changes the value function slightly, making the mortgage slightly less valuable for lower interest rates as the exogenous prepayment will happen when rates are low to the detriment of the lender, and making it lightly more valuable for high interest rates, as in this case exogenous prepayment will benefit the lender.

The plots of IO and PO parts of the mortgage show that the dynamics are very different under each of the four prepayment scenarios, as prepayments always decrease the value of the 
IO part of the mortgage (less interest will be received) and increase the value of the PO part of the mortgage (the principal will be received sooner). Note that under the interest-driven refinancing the value of the IO behaves contrary to the usual behavior of fixed income securities: the $\mathrm{IO}$ value is an increasing function of the interest rate. As interest rates decline, prepayment intensity increases making the likelihood of losing the interest payments due to prepayment greater, which wipes out any potential gains in present value of a security due to lower discount rates. As a result, IO with refinancing actually has a positive delta, in contrast to the usual negative delta of fixed income securities. One of the advantages of our analytically tractable model is in the ease of computation of the mortgage delta.

To conclude this section, we note that one of the advantages of an analytically tractable model such as the one presented in this paper is the ease of doing the sensitivity analysis, such as the analysis presented above, which greatly facilitates developing economic intuition about the problem.

\subsubsection{Model Calibration: Implied Prepayment Intensity Parameters}

To further illustrate our model, in this section we calibrate it to market data on $10 / 03 / 2005$. The credit risk of a residential mortgage is commonly estimated as that of a single-A corporate bond with the same duration (e.g., Kalotay et al. (2004)). Accordingly, to determine appropriate CIR model parameters $\theta, \kappa$, and $\sigma$, we first calibrate the CIR model to the benchmark composite single-A corporate bond yields reported by Bloomberg. The results of calibration are: $\theta=$ $0.0702, \kappa=0.147, \sigma=0.095$, and $r_{0}=0.0395$. We then compute mortgage rates without prepayment in this calibrated CIR model. The resulting term structure of mortgage rates without prepayment is plotted in Figure 4. In particular, 15-year and 30-year mortgage rates without prepayment are $5.05 \%$ and $5.335 \%$. The 15 - and 30 -year retail mortgage rate quotes were obtained from the Internet mortgage lender www.ditech.com. On 10/03/2005 the 15- and 30 -year retail mortgage rates for a conforming mortgage with no points were quoted at $5.75 \%$ and $6.125 \%$, respectively. The spreads between the idealized mortgage rates without prepayment and these retail mortgage rates are 70 basis points and 79 basis points for 15- and 30-year mortgages, respectively. These mortgage spreads can be broken up into three parts. The retail mark-up over the wholesale mortgage rates that covers the cost of marketing and origination is estimated to average $3 / 8$ of a percentage point (37.5 basis points) (e.g., web site www.mtgprofessor.com discusses the differences between retail and wholesale mortgage rates). Wholesale mortgage rates further include servicing fees that cover the cost of on-going servicing of the mortgage and are estimated to average $1 / 8$ of a percentage point (12.5 basis points) (e.g., Kalotay et al. (2004)). Finally, the remainder of the spread, net of the retail mark-up and servicing fees, is the prepayment spread we are interested in. We thus obtain current market prepayment spreads of 20 basis points and 29 basis points on 15- and 30-year mortgages, respectively. We then calculate implied prepayment intensity parameters $b$ and $\gamma$ by fitting our model to these prepayment spreads by minimizing the calibration error. The resulting implied prepayment parameters as of 10/03/2005 are: $b=0.545$ and $\gamma=15.5$, corresponding to the exogenous component of the prepayment intensity of $55 \%$ of the PSA level and an interest rate-driven refinancing component with intensity parameter $\gamma=15.5$ (the interest rate threshold $k$ in the prepayment intensity was set to $k=r_{0}-s$ with $s=0.00375$, i.e., a spread of $3 / 8$ of a percentage point ( 37.5 basis points) was deducted to account for refinancing transaction costs; this is a typical refinancing breakeven point according to the data on the web site www.mtgprofessor.com discussing mortgage 

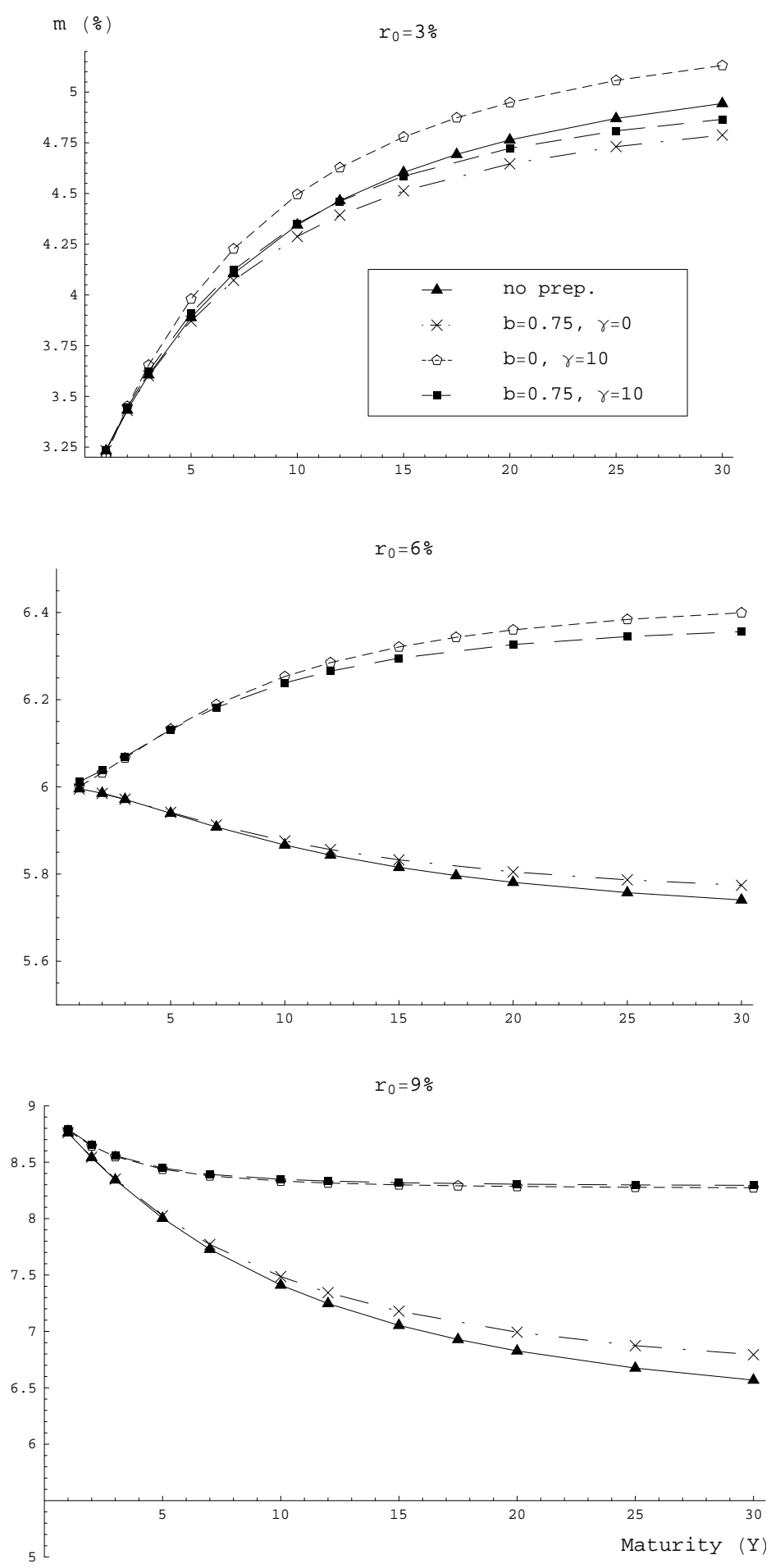

Figure 1: Mortgage rates $m=m(0, T)$ in percent as functions of maturity $T$ (in years). Model parameters: $\theta=0.06, \kappa=0.25, \sigma=0.1$. The prepayment intensity parameters and the current short rate $r_{0}$ are given on the graphs. 

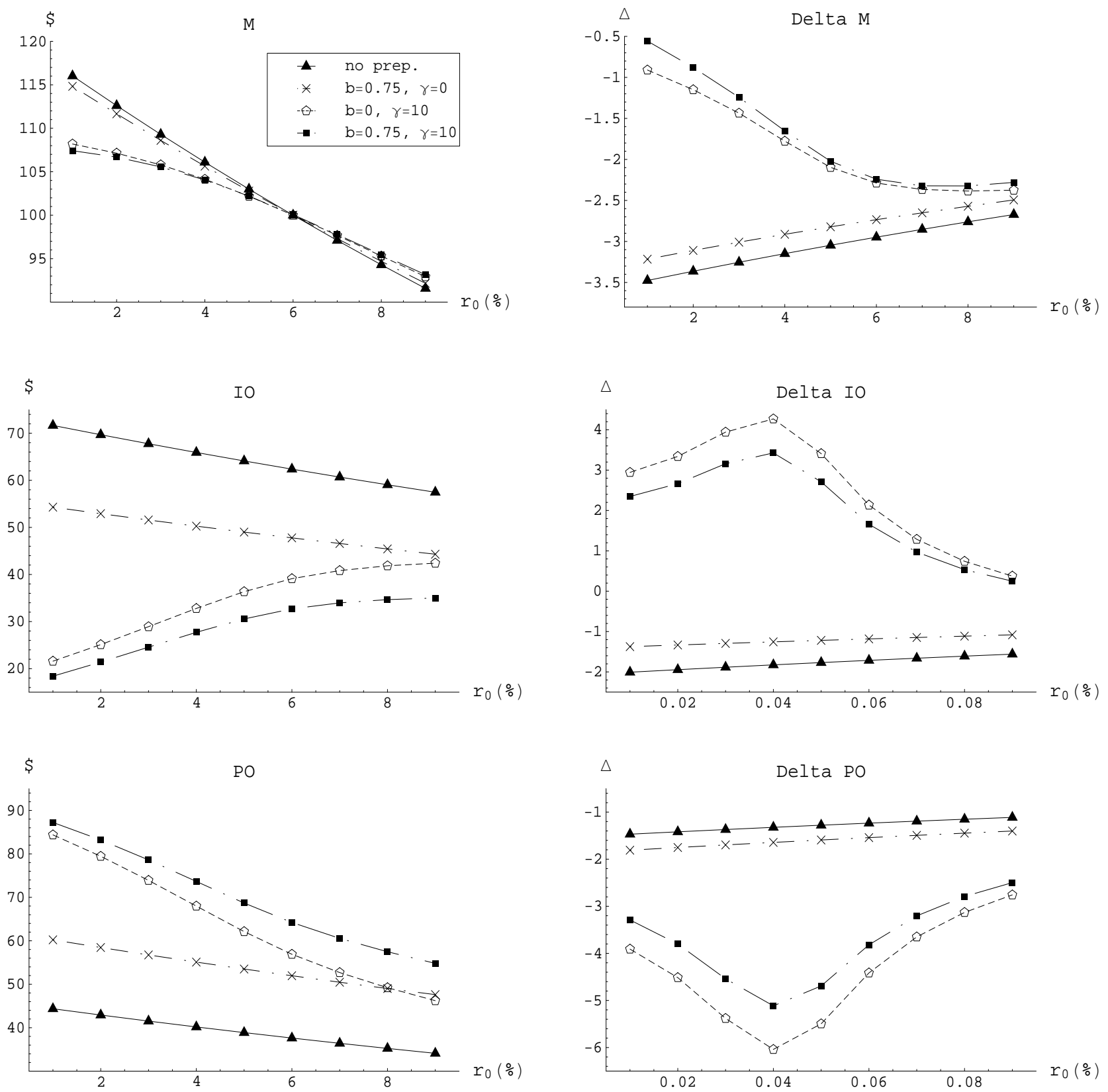

Figure 2: Mortgage values, IOs, and POs, and their deltas $(d V / d r)$ for 30-year mortgages as functions of the short rate $r$. Model parameters: $\theta=0.06, \kappa=0.25, \sigma=0.1$. The prepayment intensity parameters are given on the graphs. All four mortgages originate at par, $\$ 100$, when the short rate is $6 \%$. 


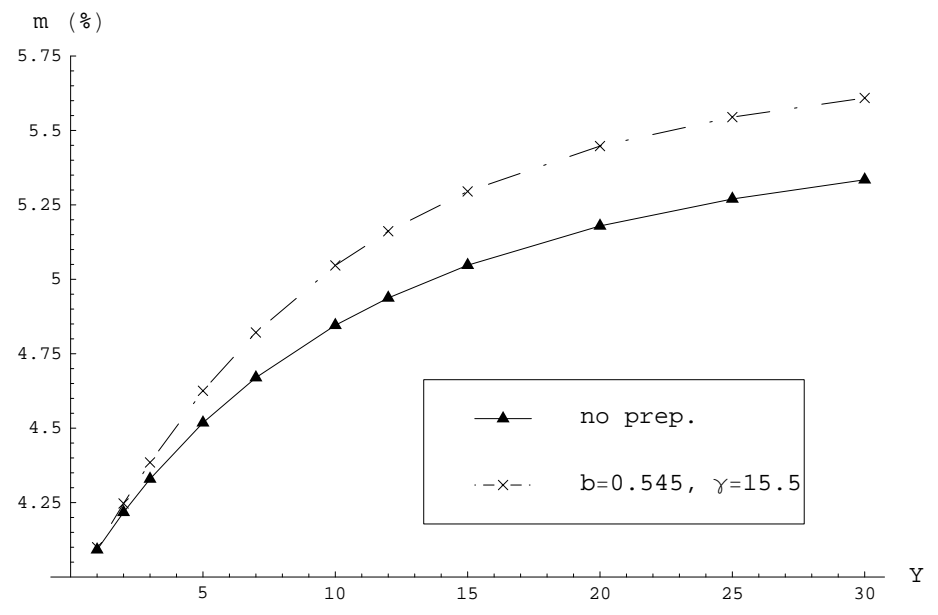

Figure 3: Results of model calibration on 10/03/2005. Mortgage rates $m=m(0, T)$ without prepayment and with the calibrated prepayment intensity as functions of maturity $T$ (in years). Calibrated CIR model parameters: $\theta=0.0702, \kappa=0.147, \sigma=0.095$. CIR short rate: $r_{0}=$ 0.0395. Calibrated prepayment intensity parameters: $b=0.545, \gamma=15.5, s=0.00375$.

refinancing). Roughly speaking, the value of the intensity parameter $\gamma=15.5$ implies that if interest rates decline by one percentage point below the threshold, there is a $15.5 \%$ chance that the mortgagor will refinance within one year (plus another $3.3 \%$ chance of prepayment coming from the exogenous refinancing of $55 \%$ of the PSA level, for a total likelihood of prepayment of $19 \%$ within a year in this scenario).

\section{Conclusion}

This paper presents an analytically tractable valuation model for residential mortgages. The random mortgage prepayment time is assumed to have an intensity represented as a sum of two terms. The first term models exogenous prepayment and is a deterministic function of time (e.g., a multiple of the PSA prepayment function). The second term models refinancing due to declining interest rates and is a multiple of the positive part of the distance between a constant threshold level (e.g., the interest rate at the mortgage origination time minus a spread to account for refinancing transaction costs) and the current interest rate. When the short rate follows a CIR diffusion, we are able to solve the model analytically and find explicit expressions for the present value of the mortgage contract, its principal-only and interest-only parts, as well as their deltas. Mortgage rates at origination are found by solving a non-linear equation. Our solution method is based on explicitly constructing an eigenfunction expansion of the pricing semigroup, a Feynman-Kac semigroup of the CIR diffusion killed at an additive functional that is a linear combination of the integral of the CIR process and an area below the constant interest rate threshold and above the interest rate sample path (the so-called area functional). A sensitivity analysis of the term structure of mortgage rates and calibration of the model to market data are presented. The eigenfunction expansion performs well computationally, with about five terms in the expansion sufficient to obtain accuracy to one basis point in determining the mortgage rate. Furthermore, the mortgage delta (sensitivity to changes in the short rate) 
is obtained analytically by direct differentiation of the eigenfunction expansion. This analytical tractability greatly facilitates conducting a detailed sensitivity analysis, model calibration, and developing economic intuition about the problem. We hope that this paper will further stimulate applications of intensity-based methodologies, that proved so successful in the credit risk area, to the important problem of mortgage modeling.

\section{A Proof of Theorem 5.1}

The proof relies on the Sturm-Liouville theory (general references are Dunford and Schwartz (1963), Levitan and Sargsjan (1975), Weidmann (1987); for finance applications see Davydov and Linetsky (2003), Gorovoi and Linetsky (2004), and Linetsky (2004a,b,c)). The problem of determining eigenvalues and eigenfunctions of the self-adjoint operator $\mathcal{G}$ in the Hilbert space $\mathcal{H}$ is equivalent to the problem of determining the eigenvalues and eigenfunctions of the associated Sturm-Liouville (SL) ordinary differential equation (ODE) problem (here $\lambda$ is a spectral parameter):

$$
-\mathcal{G} u(x)=\lambda u(x), \quad x \in(0,+\infty) .
$$

Due to the positive part function in the zero-order (killing or potential) term of $\mathcal{G}$, we need to consider this ODE in two intervals $x \in(0, k]$ and $x \in[k, \infty)$ separately. In the interval $x \in(0, k]$ we are looking for a solution $\psi_{\lambda}(x)$ of the ODE

$$
\frac{1}{2} \sigma^{2} x f^{\prime \prime}(x)+\kappa(\theta-x) f^{\prime}(x)-((1-\gamma) x+\gamma k) f(x)=-\lambda f(x)
$$

that is square-integrable with the speed density $\mathfrak{m}(x)$ on $(0, k]$ and satisfies the required boundary condition at zero:

$$
\int_{0}^{k}|f(x)|^{2} \mathfrak{m}(x) d x<\infty, \lim _{x \downarrow 0} \frac{f^{\prime}(x)}{\mathfrak{s}(x)}=0 .
$$

By a change of dependent and independent variables,

$$
z=\alpha_{1} x, f(x)=\exp \left(\frac{\left(\kappa-\rho_{1}\right) x}{\sigma^{2}}\right) u(z)
$$

this ODE reduces to the confluent hypergeometric equation (B.1). The corresponding solution is given by Eq.(5.9).

In the interval $x \in[k, \infty)$ we are looking for a solution $\phi_{\lambda}(x)$ of the ODE

$$
\frac{1}{2} \sigma^{2} x f^{\prime \prime}(x)+\kappa(\theta-x) f^{\prime}(x)-x f(x)=-\lambda f(x)
$$

that is square-integrable with the speed density $\mathfrak{m}(x)$ on $[k, \infty)$ and satisfies the required boundary condition at infinity:

$$
\int_{k}^{\infty}|f(x)|^{2} \mathfrak{m}(x) d x<\infty, \lim _{x \uparrow \infty} \frac{f^{\prime}(x)}{\mathfrak{s}(x)}=0 .
$$

By a change of dependent and independent variables,

$$
z=\alpha_{2} x, f(x)=\exp \left(\frac{\left(\kappa-\rho_{2}\right) x}{\sigma^{2}}\right) u(z),
$$


this ODE reduces to the confluent hypergeometric equation (B.1). The corresponding solution is given by Eq.(5.10).

The eigenfunctions $\varphi_{n}(x)$ of the SL problem are continuous, continuously-differentiable, square-integrable on $(0, \infty)$, satisfy the required boundary conditions at both zero and infinity, and are normalized so that $\left\|\varphi_{n}\right\|^{2}=1$. We start with the function for any $\lambda$

$$
\varphi_{n}(x)= \begin{cases}A_{\lambda} \psi_{\lambda}(x), & x \in(0, k] \\ B_{\lambda} \phi_{\lambda}(x), & x \in[k, \infty)\end{cases}
$$

with some $A_{\lambda}$ and $B_{\lambda}$ to be determined. This function is clearly square-integrable on $(0, \infty)$ and satisfies the required boundary conditions at 0 and $\infty$ due to the properties of $\psi_{\lambda}(x)$ and $\phi_{\lambda}(x)$. The continuity across $x=k$ requires

$$
A_{\lambda} \psi_{\lambda}(k)-B_{\lambda} \phi_{\lambda}(k)=0 .
$$

The continuity of the first derivative across $x=k$ requires

$$
A_{\lambda} \psi_{\lambda}^{\prime}(k)-B_{\lambda} \phi_{\lambda}^{\prime}(k)=0
$$

These two equations admit a non-trivial solution if and only if the Wronskian (5.11) vanishes (in which case they are linearly dependent). To calculate the Wronskian we used the derivatives of the confluent hypergeometric functions with respect to their argument given in Appendix B. This gives the equation for the eigenvalues $\lambda_{n}$. Finally, the overall normalization constant is found from the normalization condition $\left\|\varphi_{n}\right\|^{2}=1$, and we arrive at Eq.(5.13) for the normalized eigenfunctions.

\section{B Confluent Hypergeometric Functions}

The Kummer and Tricomi confluent hypergeometric functions $M(a, b, z)$ and $U(a, b, z)$ are solutions of the confluent hypergeometric equation

$$
z u^{\prime \prime}+(b-z) u^{\prime}-a u=0
$$

bounded at 0 and $+\infty$, respectively.

The Kummer confluent hypergeometric function $M(a, b, z)$ is defined for all $z, a \in \mathbb{C}$ and $b \in$ $\mathbb{C} \backslash\{0,-1,-2, \ldots\}$ by the confluent hypergeometric series $\left((a)_{0}:=1,(a)_{n}:=a(a+1) \ldots(a+n-1)\right.$ are the Pochhammer symbols)

$$
M(a, b, z)=\sum_{n=0}^{\infty} \frac{(a)_{n}}{(b)_{n}} \frac{z^{n}}{n !} .
$$

$M(a, b, z)$ is available as a built-in function in both Mathematica and Maple packages (in Mathematica: Hypergeometric1F1 $[\mathrm{a}, \mathrm{b}, \mathrm{z}])$. To efficiently compute the function, depending on the values of $a, b$, and $z$, these software packages use several integral representations and asymptotic expansions in addition to the series (see Abramowitz and Stegun (1972) and Slater (1960)). We used Mathematica for all calculations in this paper.

The derivative of the Kummer function with respect to $z$ is given by:

$$
\frac{d}{d z} M(a, b, z)=\frac{a}{b} M(a+1, b+1, z) .
$$


The derivative of the Kummer function with respect to its first index $a$ is given by:

$$
\frac{\partial}{\partial a} M(a, b, z)=\sum_{k=0}^{\infty} \frac{(a)_{k} \psi(a+k)}{(b)_{k} k !} z^{k}-\psi(a) M(a, b, z)
$$

where

$$
\psi(z)=\frac{\Gamma^{\prime}(z)}{\Gamma(z)}=\sum_{k=0}^{\infty}\left(\frac{1}{k}-\frac{1}{k+z-1}\right)-\gamma
$$

is the digamma function (Abramowitz and Stegun (1972)). This derivative is available in Mathematica with the call Hypergeometric1F $1^{(1,0,0)}[a, b, z]$.

The Tricomi confluent hypergeometric function $U(a, b, z)$ is defined for all $a, z \in \mathbb{C}$ and $b \in \mathbb{C} \backslash\{0, \pm 1, \pm 2, \ldots\}$ by (Slater $(1960$, p.5))

$$
U(a, b, z)=\frac{\Gamma(1-b)}{\Gamma(1+a-b)} M(a, b, z)+\frac{\Gamma(b-1)}{\Gamma(a)} x^{1-b} M(1+a-b, 2-b, z)
$$

(for $b \in \mathbb{Z}, U(a, b, z)$ is defined as a $\operatorname{limit}_{\epsilon \rightarrow 0} U(a, b+\epsilon, z)$; see Slater (1960)). It is available in Mathematical with the call HypergeometricU[a, b, z] .

The derivative of the Tricomi function with respect to $z$ is given by:

$$
\frac{d}{d z} U(a, b, z)=-a U(a+1, b+1, z)
$$

The derivative with respect to its first index $a$ is (this derivative is available in Mathematica with the call Hypergeometric $\mathrm{U}^{(1,0,0)}[a, b, z]$ :

$$
\begin{gathered}
\frac{\partial}{\partial a} U(a, b, z)=\frac{\Gamma(b-1) z^{1-b}}{\Gamma(a)} \sum_{k=0}^{\infty} \frac{\psi(a-b+k+1)(a-b+1)_{k} z^{k}}{k !(2-b)_{k}} \\
+\frac{\Gamma(1-b)}{\Gamma(a-b+1)} \sum_{k=0}^{\infty} \frac{\psi(a+k)(a)_{k} z^{k}}{k !(b)_{k}}-\{\psi(a)+\psi(a-b+1)\} U(a, b, z) .
\end{gathered}
$$

\section{CIR Annuities}

Here we give explicit expressions for continuous and discrete annuities entering Eqs.(2.3) and (2.6) in the CIR model. The Feynman-Kac pricing semigroup for the CIR process $\left\{r_{t}, t \geq 0\right\}$ with discounting (killing) at the rate $r_{t}$ has the infinitesimal generator

$$
\mathcal{G} f(r)=\frac{1}{2} \sigma^{2} r f^{\prime \prime}(r)+\kappa(\theta-r) f^{\prime}(r)-r f(r) .
$$

Its eigenvalues and normalized eigenfunctions in $L^{2}((0, \infty), \mathfrak{m})$, where $\mathfrak{m}$ is the speed density of the CIR process, are given by (Davydov and Linetsky (2003); note that here it is convenient for us to label the eigenvalues starting from zero, $n=0,1, \ldots$ in contrast to the operator (5.5) with prepayment, for which we label the eigenvalues starting from $n=1$ ):

$$
\lambda_{n}=\rho n+\frac{\beta}{2}(\rho-\kappa), \text { where } \rho:=\sqrt{\kappa^{2}+2 \sigma^{2}},
$$




$$
\varphi_{n}(r)=\sqrt{\frac{\sigma^{2} n !}{2 \Gamma(\beta+n)}}\left(\frac{2 \rho}{\sigma^{2}}\right)^{\frac{\beta}{2}} e^{-\frac{(\rho-\kappa) r}{\sigma^{2}}} L_{n}^{(\beta-1)}\left(\frac{2 \rho r}{\sigma^{2}}\right),
$$

where $L_{n}^{(\alpha)}(x)$ are the generalized Laguerre polynomials. The eigenfunction expansion of the unit-face zero-coupon bond value function is in the form

$$
B(r ; 0, T)=\sum_{n=0}^{\infty} c_{n} e^{-\lambda_{n} T} \varphi_{n}(r)
$$

with the expansion coefficients (Davydov and Linetsky (2003))

$$
c_{n}=(-1)^{n} \sqrt{\frac{2 \Gamma(\beta+n)}{\sigma^{2} n !}}\left(\frac{\sigma \sqrt{2 \rho}}{\rho+\kappa}\right)^{\beta}\left(\frac{\rho-\kappa}{\kappa+\rho}\right)^{n} .
$$

Applying the well-known identity for the generating function of Laguerre polynomials (Eq.(17) in Erdelyi (1953, p.189))

$$
\sum_{n=0}^{\infty} z^{n} L_{n}^{(\alpha)}(x)=(1-z)^{-\alpha-1} \exp \left\{\frac{x z}{z-1}\right\},
$$

the eigenfunction expansion for the CIR bond price collapses to the CIR bond pricing formula:

$$
B(r ; 0, T)=A(T) e^{-B(T) r}
$$

where

$$
B(T)=\frac{2\left(e^{\rho T}-1\right)}{(\rho+\kappa)\left(e^{\rho T}-1\right)+2 \rho}, \quad A(T)=\left(\frac{2 \rho e^{(\kappa+\rho) T / 2}}{(\rho+\kappa)\left(e^{\rho T}-1\right)+2 \rho}\right)^{\beta} .
$$

Thus, we have two alternative representations of the zero-coupon bond value function: as the eigenfunction expansion (C.1) and the closed-form formula (C.2). ${ }^{3}$ Generally, the closed-form formula (C.2) is computationally more advantageous. However, to compute the continuous annuity, we need to integrate the zero-coupon bond value function with respect to maturity. The integral of (C.2) does not appear to be analytically tractable directly. However, the eigenfunction expansion (C.1) can be integrated to yield the value function of the continuous annuity:

$$
A_{c}(r ; 0, T)=\int_{0}^{T} B(r ; 0, t) d t=\sum_{n=0}^{\infty} \frac{1-e^{-\lambda_{n} T}}{\lambda_{n}} c_{n} \varphi_{n}(r) .
$$

Similarly, the sum in $k$ in the discrete annuity formula is calculated in closed form to yield $(T=N \Delta)$ :

$$
A_{\Delta}(r ; 0, T)=\Delta \sum_{k=1}^{N} B(r ; 0, k \Delta)=\sum_{n=0}^{\infty}\left(\sum_{k=1}^{N} e^{-\lambda_{n} k \Delta} \Delta\right) c_{n} \varphi_{n}(r)=\sum_{n=0}^{\infty} \frac{\Delta\left(1-e^{-\lambda_{n} T}\right)}{e^{\lambda_{n} \Delta}-1} c_{n} \varphi_{n}(r) .
$$

In the limit $\Delta \rightarrow 0$ the discrete annuity converges to the continuous annuity formula.

\footnotetext{
${ }^{3}$ We note that similar expressions to the CIR bond pricing formula were obtained by Wong (1964), Eq.(53) and Pitman and Yor (1982) in different contexts.
} 


\section{References}

[1] Abramowitz, M., and I.A. Stegun, 1972, Handbook of Mathematical Functions, Dover, New York.

[2] Bielecki, T., and M. Rutkowski, 2002, Credit Risk: Modeling, Valuation and Hedging, Springer, Berlin.

[3] Borodin, A.N., and P. Salminen, 2002, Handbook of Brownian Motion, 2nd Ed., Birkhauser, Boston.

[4] Carr, P., and V. Linetsky, 2000, "The Valuation of Executive Stock Options in an IntensityBased Framework," European Finance Review, 4, 211-230.

[5] Cizek P., W. Hardle, and R. Weron, 2005, Statistical Tools for Finance and Insurance, Springer, Berlin.

[6] Cox, J.C., J.E. Ingersoll and S.A. Ross, 1985, "A Theory of the Term Structure of Interest Rates," Econometrica, 53, 385-407.

[7] Davydov, D., and V. Linetsky, 2003, "Pricing Options on Scalar Diffusions: An Eigenfunction Expansion Approach," Operations Research, 51, 185-209.

[8] Deng, Y., J.M. Quigley, and R. Van Order, 2000, "Mortgage Termination, Heterogeneity, and the Exercise of Mortgage Options," Econometrica, 68, 275-307.

[9] Duffie, D., 1996, Dynamic Asset Pricing, 1st Ed., Princeton University Press, Princeton, NJ.

[10] Duffie, D., and K. Singleton, 1999, "Modeling Term Structures of Defaultable Bonds," Review of Financial Studies, 12, 687-720.

[11] Duffie, D., and K. Singleton, 2003, Credit Risk, Princeton University Press, Princeton, NJ.

[12] Dunford, N. and J. Schwartz, 1963, Linear Operators. Part II: Spectral Theory (Self-Adjoint Operators in Hilbert Spaces), Wiley, NJ.

[13] Dunn, K.,B., and J.J. McConnel, 1981a, "A Comparison of Alternative Models for Pricing GNMA Mortgage-Backed Securities," Journal of Finance, 36, 471-484.

[14] Dunn, K.,B., and J.J. McConnel, 1981b, "Valuation of GNMA Mortgage-Backed Securities," Journal of Finance, 36, 599-616.

[15] Elliot, R.J., M. Jeanblanc, and M. Yor, 2000, "On Models of Default Risk," Mathematical Finance, 10, 179-196.

[16] Erdelyi, A., 1953, Higher Transcendental Functions, Vol.II, MacGraw-Hill, New York.

[17] Feller, W., 1951, "Two Singular Diffusion Problems," Annals of Mathematics, 54, 173-82.

[18] Gorovoi V. and Linetsky V., 2004, "Black's Model of Interest Rates as Options, Eigenfunction Expansions and Japanese Interest Rates", Mathematical Finance, 14, 49-78. 
[19] Goncharov, Y., 2003, "Mathematical Theory of Mortgage Modeling," Ph.D. dissertation, University of Illinois at Chicago (http://www.math.fsu.edu/ goncharo/).

[20] Goncharov, Y., 2004, "On the Mortgage Rates Implied by the Option-Based and Empirical Approaches," Proceedings of the Hawaii International Conference on Statistics, Honolulu.

[21] Goncharov, Y., 2006, "An Intensity-Based Approach to the Valuation of Mortgage Contracts and Computation of the Endogenous Mortgage Rate," International Journal of Theoretical and Applied Finance, to appear.

[22] Jarrow, R., D. Lando, F. Yu, 2005, "Default Risk and Diversification: Theory and Empirical Implications," Mathematical Finance, 15, 1-26.

[23] Jarrow, R., and S. Turnbull, 1995, "Pricing Derivatives on Financial Securities Subject to Credit Risk," Journal of Finance, March, 53-85.

[24] Jeanblanc, M., M. Yor, and M. Chesney, 2006, Mathematical Methods for Financial Markets, Springer, Berlin, to appear.

[25] Kagraoka, Y., 2002, "The OAS Approach and the Martingale Measure for Mortgage Prepayment," Working Paper, Musahi University.

[26] Kalotay, A., D. Yang, and F.J. Fabozzi, 2004, "An Option-Theoretic Prepayment Model for Mortgages and Mortgage-Backed Securities," International Journal of Theoretical \& Applied Finance, 7, 949-978.

[27] Kau, J.B., D.C. Keenan, and A.A. Smurov, 2004, "Reduced Form Mortgage Valuation," working paper, University of Georgia.

[28] Lando, D., 2004, Credit Risk Modeling, Princeton University Press, Princeton, NJ.

[29] Levitan, B.M., and I.S. Sargsjan, 1975, Introduction to Spectral Theory, American Mathematical Society, Providence, RI.

[30] Linetsky, V., 2004a, "Lookback Options and Diffusion Hitting Times: A Spectral Expansion Approach," Finance and Stochastics, 8 (3), 373-398.

[31] Linetsky, V., 2004b, "Computing Hitting Time Densities for CIR and OU Diffusions: Applications to Mean-Reverting Models," Journal of Computational Finance, 7 (4), 1-22.

[32] Linetsky, V., 2004c, "The Spectral Decomposition of the Option Value," International Journal of Theoretical \& Applied Finance, 7 (3), 337-384.

[33] Longstaff, F.A., 2005, "Borrower Credit and the Valuation of Mortgage-backed Securities," Real Estate Economics, 33(4), 619-661.

[34] Merton, R., 1976, "Option Pricing When Underlying Stock Returns Are Discontinuous," Journal of Financial Economics, 3, 125-144.

[35] Perman, M. and J. Wellner, 1996, "On the Distribution of Brownian Areas," Annals of Applied Probability, 6, 1091-1111. 
[36] Pitman J., and M. Yor, 1982, "A Decomposition of Bessel Bridges," Zeit. Wahrsch. Geb., $59,425-457$.

[37] Pliska, S., 2005, "Optimal Mortgage Refinancing with Endogenous Mortgage Rates: an Intensity Based, Equilibrium Approach," Working Paper, University of Illinois at Chicago.

[38] Pliska, S., 2006, "Mortgage Valuation and Optimal Refinancing," in Stochastic Finance, Proceedings of the 2004 Conference in Lisbon, Portugal, Edited by A. Shiryaev et al., Springer.

[39] Schwartz, E., and W. Torous, 1989, "Prepayment and the Valuation of Mortgage-Backed Securities," Journal of Finance, 44, 375-392.

[40] Schwartz, E., and W. Torous, 1992, "Prepayment, Default, and the Valuation of Mortgage Pass-through Securities," Journal of Business, 65, 221-239.

[41] Schwartz, E., and W. Torous, 1993, "Mortgage Prepayment and Default Decisions: A Poisson Regression Approach," Journal of the American Real Estate and Urban Economics Association, 21, 431-449.

[42] Slater, L.J., 1960, Confluent Hypergeometric Functions, Cambridge University Press.

[43] Stanton, R., 1995, "Rational Prepayment and the Valuation of Mortgage-Backed Securities," Review of Financial Studies, 8, 677-708.

[44] Weidmann, J., 1987, Spectral Theory of Ordinary Differential Operators, Lecture Notes in Mathematics, Vol. 1258, Springer, Berlin.

[45] Wong, E., 1964, "The Construction of A Class of Stationary Markoff Processes," In: Bellman, R. (Ed.), Sixteenth Symposium in Applied Mathematics - Stochastic Processes in Mathematical Physics and Engineering, American Mathematical Society, Providence, RI, pp. 264-276. 